\title{
TEMPO-Mediated Aza-Diels-Alder Reaction: Synthesis of Tetrahydropyridazines Using Ketohydrazones and Olefins
}

\author{
Xiu-Long Yang, Xie-Xue Peng, Fei Chen, and Bing Han* \\ State Key Laboratory of Applied Organic Chemistry, College of Chemistry and Chemical \\ Engineering, Lanzhou University, Lanzhou, Gansu, 730000, People's Republic of China \\ hanb@lzu.edu.cn
}

\section{Supporting Information}

- Contents

page

- General

- General experimental procedure

- References

- Analytical data for products

- ORTEP plot for compound $3 y$

- Copies of ${ }^{1} \mathrm{H}$ and ${ }^{13} \mathrm{C}$ spectra 


\section{General:}

All reagents were purchased from commercial suppliers and used without further purification. Flash chromatography was carried out with silica gel (200-300 mesh). Analytical TLC was performed with silica gel GF254 plates, and the products were visualized by UV detection. ${ }^{1} \mathrm{H}$ NMR and ${ }^{13} \mathrm{C}$ NMR (400 MHz, and $100.6 \mathrm{MHz}$ respectively) spectra were recorded in $\mathrm{CDCl}_{3}$ and DMSO- $d_{6}$. Chemical shifts $(\delta)$ are reported in ppm using TMS as internal standard and spin-spin coupling constants $(J)$ are given in $\mathrm{Hz}$. El-MS spectra were measured on a TRACE DSQ spectrometer by direct inlet at $70 \mathrm{eV}$. The high resolution mass spectra (HRMS) were measured on a Bruker Daltonics APEX II 47e spectrometer by ESI. Data collections for crystal structure were performed at room temperature $(293 \mathrm{~K})$ using MoK $\alpha$ radiation on a Bruker APEXII diffractometer. Integration of the frames and data reduction was carried out using SAINT. The structure was solved by direct methods using SHELXS-97.

\section{General experimental procedure:}

Table S1. Involved hydrazones

(n)


Table S2. Involved olefins

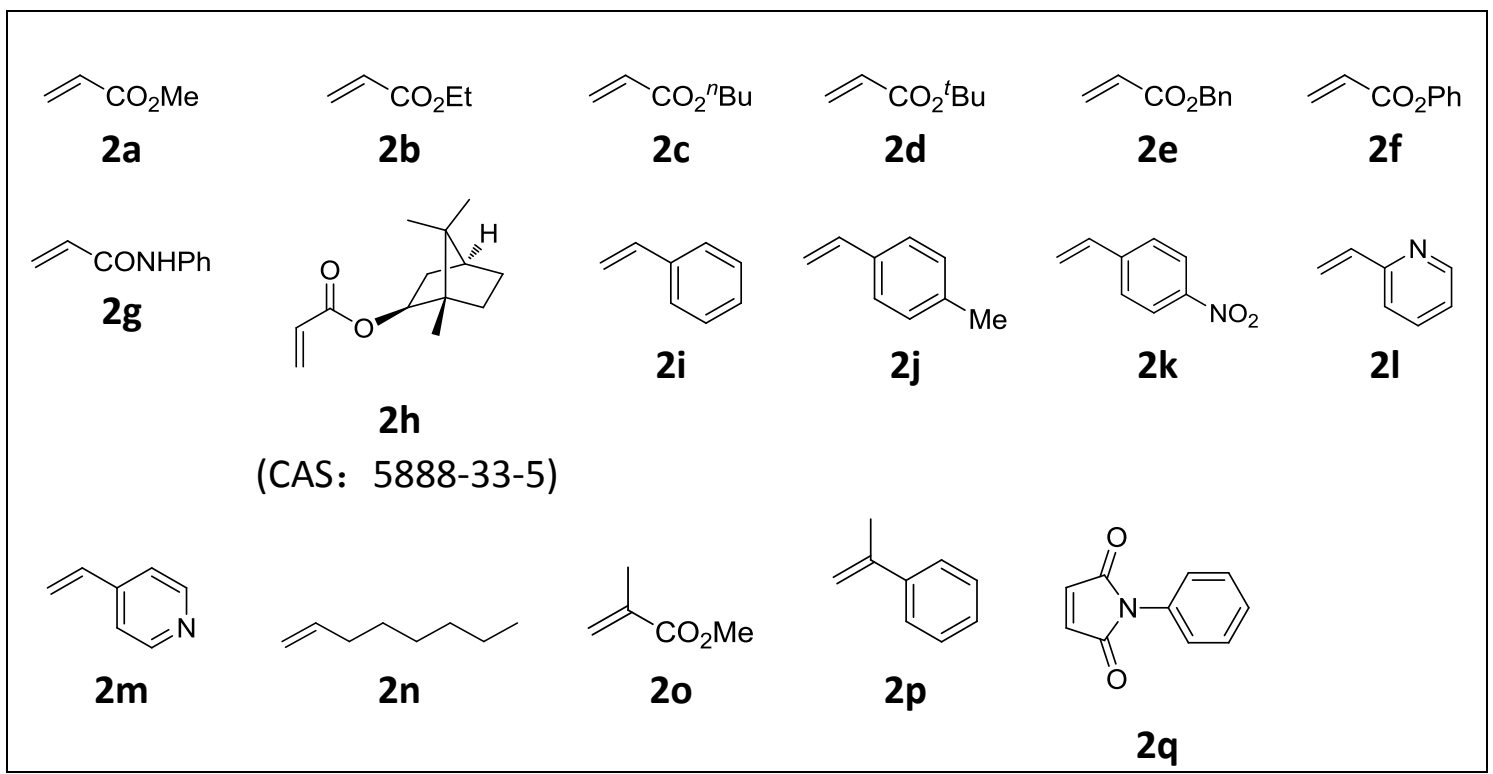

\section{General procedure for the synthesis of ketohydrazones}

Method A: for the synthesis of aromatic and aliphatic hydrazones $\mathbf{1 a}-\mathbf{1} \mathbf{h}$ and $\mathbf{1} \mathbf{j}-\mathbf{1} \mathbf{q}^{\mathbf{1}, \mathbf{2}}$

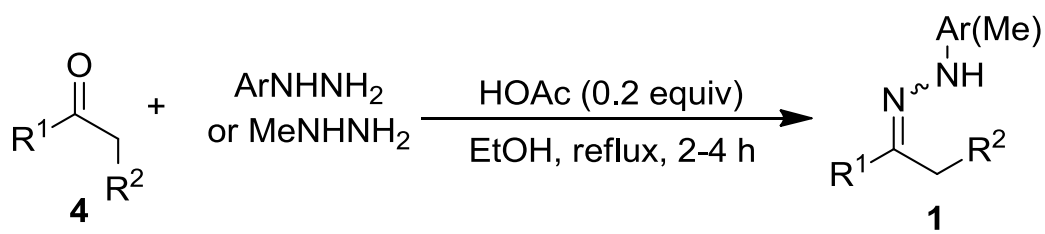

The acetic acid (0.2 equiv) was added to a mixture of hydrazines (1.05 equiv) and ketones (1.0 equiv) in anhydrous ethanol, and then heated at reflux for 2-4 hours under Ar until the condensation was completed (monitored by TLC). The mixture was cooled in ice and the precipitate was filtered off and rinsed with cooled ethanol and petroleum ether.

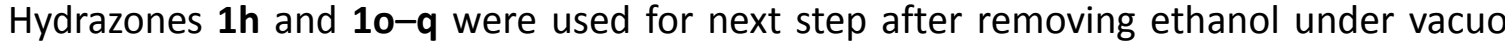
without further purification.

Notes: Due to the autoxidation of some hydrazones, the fresh prepared hydrazones were used immediately. ${ }^{1 \mathrm{c}}$

Method B: for the synthesis of benzoyl hydrazone $1 \mathbf{i}^{3}$ 


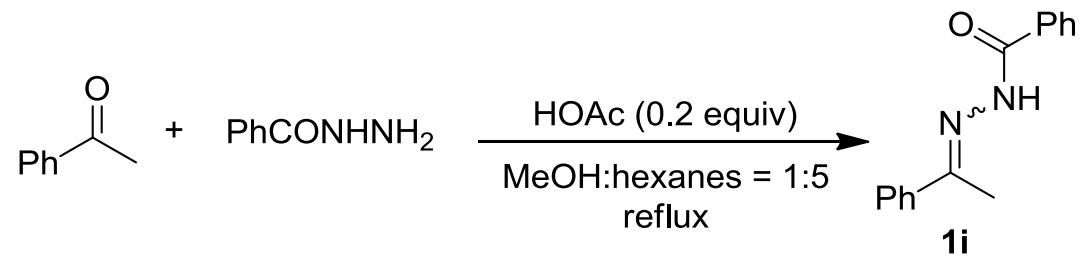

Benzoylhydrazine (408 mg, $3 \mathrm{mmol}, 1.5$ equiv) was added to a stirred solution of acetophenone ( $240 \mathrm{mg}, 2 \mathrm{mmol}, 1$ equiv) and glacial acetic acid ( $24 \mathrm{mg}, 0.4 \mathrm{mmol}, 0.2$ equiv) in a mixed solvent of methanol/hexanes $(1: 5,5 \mathrm{~mL})$. The resulting suspension was heated to reflux until the ketone was consumed completely. The solvent was evaporated and purification of the crude product by flash column chromatography afforded hydrazone $1 \mathbf{i}$ (381 $\mathrm{mg}, 80 \%)$ as a white solid.

\section{General procedure for Aza-Diels-Alder reaction of hydrazones and alkenes}

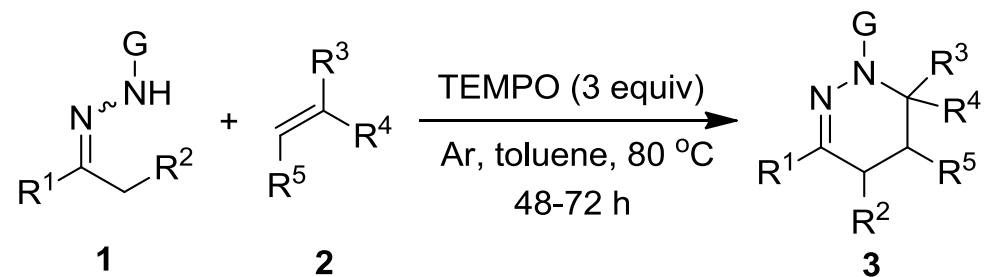

A mixture of ketohydrazones $(0.5 \mathrm{mmol})$, alkenes $(5.0 \mathrm{mmol})$ and TEMPO $(1.5 \mathrm{mmol})$ was placed in a $10 \mathrm{~mL}$ oven-dried Schlenk tube in toluene $(1 \mathrm{~mL})$ and stirred at $80{ }^{\circ} \mathrm{C}$ under argon for $48-72 \mathrm{~h}$. When hydrazones were consumed completely as monitored by TLC, the mixture was diluted with EtOAc. The solvent was then removed under vacuo. The residue was purified with chromatography column on silica gel (gradient eluent of EtOAc/petroleum ethers: $1 / 20$ to $1 / 5$ ) to give the corresponding products 3 in yields listed in Scheme 2 and Scheme 3. The identity and purity of the product was confirmed by ${ }^{1} \mathrm{H}$ and ${ }^{13} \mathrm{C}$ NMR spectroscopic analysis.

General procedure for the one-pot Aza-Diels-Alder reaction of phenylhydrazines, ketones and alkenes

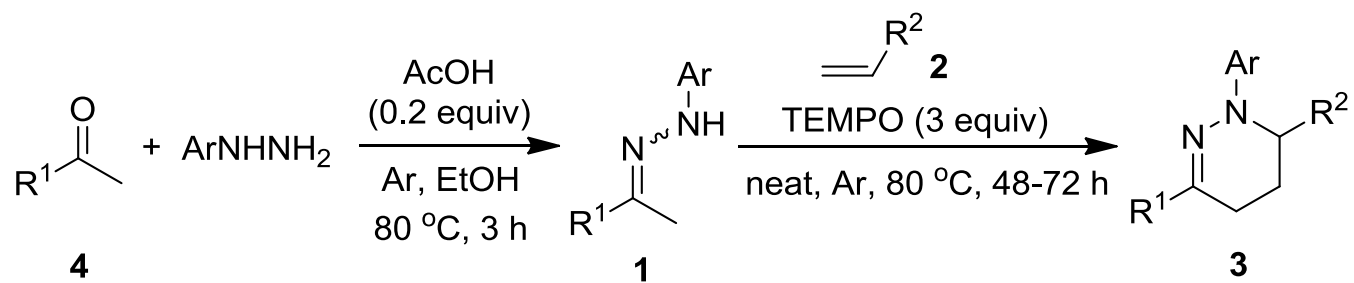

The mixture of ketone $(1 \mathrm{mmol})$, phenylhydrazine $(1.05 \mathrm{mmol})$ and acetic acid $(0.2 \mathrm{mmol})$ 
were refluxed in EtOH $(2.0 \mathrm{~mL})$ under argon until the condensation was completed (monitored by TLC, about $3 \mathrm{~h}$ ). After removing the solvent, TEMPO $(3.0 \mathrm{mmol}$ ) and alkenes $(10.0 \mathrm{mmol})$ were added, and the mixture was stirred at $80{ }^{\circ} \mathrm{C}$ under argon for 48-72 h. When hydrazones were consumed completely as monitored by TLC, the mixture was diluted with EtOAc. The solvent was then removed under vacuo. The residue was purified with chromatography column on silica gel (gradient eluent of EtOAc/petroleum ethers: $1 / 20$ to $1 / 5$ ) to give the corresponding products 3 in yields listed in Scheme 4. The identity and purity of the product was confirmed by ${ }^{1} \mathrm{H}$ and ${ }^{13} \mathrm{C}$ NMR spectroscopic analysis.

\section{References:}

(1) (a) Zhang, G.-W.; Miao, J.-M.; Zhao, Y.; Ge, H.-B. Angew. Chem., Int. Ed. 2012, 51, 8318-8321. (b) Hu, J.-T.; Xu, H.-C.; Nie, P.-J.; Xie, X.-B.; Nie, Z.-X.; Rao, Y. Chem. - Eur. J. 2014, 20, 3932-3938. (c) Harej, M.; Dolenc, D. J. Org. Chem. 2007, 72, 7214-7221.

(2) Klein, J. J.; Hecht, S. Org. Lett. 2012, 14, 330-333.

(3) (a) Wu, P.-L.; Peng, S.-Y.; Magrath, J. Synthesis 1995, 435-438. (b) Berger, R.; Duff, K.; Leighton, J. L. J. Am. Chem. Soc. 2004, 126, 5686-5687.

\section{Analytical data for products:}

methyl 2,6-diphenyl-2,3,4,5-tetrahydropyridazine-3-carboxylate (3a)<smiles>CC(=O)C1CCC(c2ccccc2)=NN1c1ccccc1</smiles>

White solid; (119 mg, 81\%); mp: $99-100{ }^{\circ} \mathrm{C} ;{ }^{1} \mathrm{H}$ NMR (400 MHz, $\mathrm{CDCl}_{3}$ ): $\delta 7.81$ (d, J= 7.6 $\mathrm{Hz}, 2 \mathrm{H}), 7.24-7.39(\mathrm{~m}, 7 \mathrm{H}), 6.92(\mathrm{~m}, 1 \mathrm{H}), 4.77(\mathrm{~d}, J=4.4 \mathrm{~Hz}, 1 \mathrm{H}), 3.70(\mathrm{~s}, 3 \mathrm{H}), 2.75(\mathrm{dd}, J$ $=17.2,4.8 \mathrm{~Hz}, 1 \mathrm{H}), 2.55(\mathrm{~m}, 1 \mathrm{H}), 2.45(\mathrm{~m}, 1 \mathrm{H}), 2.16(\mathrm{~m}, 1 \mathrm{H}) ;{ }^{13} \mathrm{C} \mathrm{NMR}\left(100.6 \mathrm{MHz}, \mathrm{CDCl}_{3}\right)$ : $\delta 172.0,146.8,139.3,138.3,129.0,128.2,127.8,124.7,120.3,113.5,54.0,52.6,20.8$, 18.7; MS m/z (relative intensity, \%): 294 (28.9), 249 (12.4), 235 (100), 143 (13.7), 104 (7.0), 77 (21.3); ESI-HRMS: $m / z$ Calcd for $\mathrm{C}_{18} \mathrm{H}_{18} \mathrm{~N}_{2} \mathrm{O}_{2}+\mathrm{H}^{+}: 295.1441$, found 295.1434. 
<smiles>COC(=O)C1CCC(c2ccccc2)=NN1c1ccc(C)cc1</smiles>

White solid; (128 mg, 83\%); mp: $\left.139-140{ }^{\circ} \mathrm{C} ;{ }^{1} \mathrm{H} \mathrm{NMR} \mathrm{(400} \mathrm{MHz,} \mathrm{CDCl}_{3}\right): \delta 7.80$ (d, $J=$ $8.4 \mathrm{~Hz}, 2 \mathrm{H}), 7.37(\mathrm{t}, J=8.0,7.2 \mathrm{~Hz}, 2 \mathrm{H}), 7.28(\mathrm{~m}, 1 \mathrm{H}), 7.20(\mathrm{~d}, J=8.4 \mathrm{~Hz}, 2 \mathrm{H}), 7.11(\mathrm{~d}, J=$ $8.4 \mathrm{~Hz}, 2 \mathrm{H}), 4.75(\mathrm{~d}, J=4.4 \mathrm{~Hz}, 1 \mathrm{H}), 3.70(\mathrm{~s}, 3 \mathrm{H}), 2.74(\mathrm{~m}, 1 \mathrm{H}), 2.55(\mathrm{~m}, 1 \mathrm{H}), 2.44(\mathrm{~m}, 1 \mathrm{H})$, $2.30(\mathrm{~s}, 3 \mathrm{H}), 2.16(\mathrm{~m}, 1 \mathrm{H}) ;{ }^{13} \mathrm{C}$ NMR $\left(100.6 \mathrm{MHz} \mathrm{CDCl}_{3}\right): \delta 172.1,144.7,138.7,138.5$, 129.6, 128.2, 127.7, 124.6, 113.6, 54.1, 52.5, 20.8, 20.4, 18.6; MS $\mathrm{m} / \mathrm{z}$ (relative intensity, \%): 308 (36.2), 250 (19.4), 249 (100), 143 (7.7), 91 (16.4), 77 (10.2); ESI-HRMS: $\mathrm{m} / \mathrm{z}$ Calcd for $\mathrm{C}_{19} \mathrm{H}_{20} \mathrm{~N}_{2} \mathrm{O}_{2}+\mathrm{H}^{+}: 309.1598$, found 309.1588 .

methyl 2-(4-methoxyphenyl)-6-phenyl-2,3,4,5-tetrahydropyridazine-3-carboxylate (3c)<smiles>COc1ccc(N2N=C(c3ccccc3)CCC2C(C)=O)cc1</smiles>

White solid; (105 mg, 65\%); mp: $126-127{ }^{\circ} \mathrm{C} ;{ }^{1} \mathrm{H}$ NMR (400 MHz, $\mathrm{CDCl}_{3}$ ): $\delta 7.78$ (d, $J=7.2$ $\mathrm{Hz}, 2 \mathrm{H}), 7.35(\mathrm{t}, J=7.6 \mathrm{~Hz}, 2 \mathrm{H}), 7.27(\mathrm{~m}, 1 \mathrm{H}), 7.23(\mathrm{~m}, 2 \mathrm{H}), 6.88(\mathrm{~d}, J=8.8 \mathrm{~Hz}, 2 \mathrm{H}), 4.70(\mathrm{~d}$, $J=4.4 \mathrm{~Hz}, 1 \mathrm{H}), 3.77(\mathrm{~s}, 3 \mathrm{H}), 3.69(\mathrm{~s}, 3 \mathrm{H}), 2.70(\mathrm{~m}, 1 \mathrm{H}), 2.53(\mathrm{~m}, 1 \mathrm{H}), 2.43(\mathrm{~m}, 1 \mathrm{H}), 2.18(\mathrm{~m}$, 1H); ${ }^{13} \mathrm{C}$ NMR $\left(100.6 \mathrm{MHz}, \mathrm{CDCl}_{3}\right): \delta 172.1,154.0,141.2,138.44,138.41,128.2,127.6$, 124.5, 115.1, 114.4, 55.6, 54.5, 52.5, 20.8, 18.5; MS m/z (relative intensity, \%): 325 (11.7), 324 (55.2), 265 (100), 122 (364), 92 (5.8), 77 (13.2); ESI-HRMS: m/z Calcd for $\mathrm{C}_{19} \mathrm{H}_{20} \mathrm{~N}_{2} \mathrm{O}_{3}+\mathrm{H}^{+}:$325.1547, found 325.1541.

methyl 2-(4-chlorophenyl)-6-phenyl-2,3,4,5-tetrahydropyridazine-3-carboxylate (3d) 
<smiles>CC(=O)C1CCC(c2ccccc2)=NN1c1ccc(Cl)cc1</smiles>

White solid; (96 mg, 58\%); mp: $123-124{ }^{\circ} \mathrm{C} ;{ }^{1} \mathrm{H}$ NMR (400 MHz, $\mathrm{CDCl}_{3}$ ): $\delta 7.78$ (d, $J=7.2$ $\mathrm{Hz}, 2 \mathrm{H}), 7.38(\mathrm{t}, J=7.2 \mathrm{~Hz}, J=7.62 \mathrm{~Hz}, 2 \mathrm{H}), 7.21-7.32(\mathrm{~m}, 5 \mathrm{H}), 4.72(\mathrm{~d}, J=3.6 \mathrm{~Hz}, 1 \mathrm{H})$, $3.71(\mathrm{~s}, 3 \mathrm{H}), 2.75(\mathrm{~m}, 1 \mathrm{H}), 2.56(\mathrm{~m}, 1 \mathrm{H}), 2.44(\mathrm{~m}, 1 \mathrm{H}), 2.17(\mathrm{~m}, 1 \mathrm{H}) ;{ }^{13} \mathrm{C}$ NMR $(100.6 \mathrm{MHz}$, $\left.\mathrm{CDCl}_{3}\right): \delta 171.6,145.5,140.2,138.1,128.9,128.3,128.1,125.2,124.8,114.7,54.0,52.6$, 20.7, 18.6; MS m/z (relative intensity, \%): 330 (14.1), 328 (44.8), 271 (31.4), 269 (18.1), 234 (15.6), 143 (19.1), 77 (11.4); ESI-HRMS: $m / z$ Calcd for $\mathrm{C}_{18} \mathrm{H}_{17} \mathrm{ClN}_{2} \mathrm{O}_{2}+\mathrm{H}^{+}: 329.1051$, found 329.1047 .

methyl-6-phenyl-2-(4-(trifluoromethyl)phenyl)-2,3,4,5-tetrahydropyridazine-3-carboxyl ate (3e)<smiles>CC(=O)C1CCC(c2ccccc2)=NN1c1ccc(C(F)(F)F)cc1</smiles>

White solid; (100 mg, 55\%); mp: $118-120{ }^{\circ} \mathrm{C} ;{ }^{1} \mathrm{H}$ NMR (400 MHz, $\mathrm{CDCl}_{3}$ ): $\delta 7.80$ (d, $J=$ $7.6 \mathrm{~Hz}, 2 \mathrm{H}), 7.53$ (d. $J=8.8 \mathrm{~Hz}, 2 \mathrm{H}), 7.30-7.40(\mathrm{~m}, 5 \mathrm{H}), 4.78(\mathrm{~d}, J=3.6 \mathrm{~Hz}, 1 \mathrm{H}), 3.71(\mathrm{~s}$, 3H), $2.76(\mathrm{dd}, J=17.6,4.4 \mathrm{~Hz}, 1 \mathrm{H}), 2.58(\mathrm{~m}, 1 \mathrm{H}), 2.45(\mathrm{~m}, 1 \mathrm{H}), 2.16(\mathrm{~m}, 1 \mathrm{H}) ;{ }^{13} \mathrm{C} N M R$ $\left(100.6 \mathrm{MHz}, \mathrm{CDCl}_{3}\right): \delta 171.3,149.1,141.5,137.8,128.4,128.3,126.31\left(q,{ }^{3} J_{C, F}=3.6 \mathrm{~Hz}\right)$, 126.31, 124.9, $124.72\left(q,{ }^{1} J_{C, F}=269.1 \mathrm{~Hz}\right), 121.70\left(q,{ }^{2} J_{C, F}=32.4 \mathrm{~Hz}\right), 112.8,53.7,52.7,20.6$, 18.7; MS m/z (relative intensity, \%): 363 (8.8), 362 (44.4), 304 (17.8), 303 (100), 143(15.4), 77 (10.6); ESI-HRMS: $\mathrm{m} / z$ Calcd for $\mathrm{C}_{19} \mathrm{H}_{17} \mathrm{~F}_{3} \mathrm{~N}_{2} \mathrm{O}_{2}+\mathrm{H}^{+}: 363.1315$, found 363.1302.

methyl 2-(4-cyanophenyl)-6-phenyl-2,3,4,5-tetrahydropyridazine-3-carboxylate (3f) 
<smiles>CC(=O)C1CCC(c2ccccc2)=NN1c1ccc(C#N)cc1</smiles>

Yellow solid; (80 mg, 50\%); mp: $113-114{ }^{\circ} \mathrm{C} ;{ }^{1} \mathrm{H}$ NMR (400 MHz, $\mathrm{CDCl}_{3}$ ): $\delta 7.80$ (d, J = 7.2 $\mathrm{Hz}, 2 \mathrm{H}), 7.56(\mathrm{~d}, J=8.8 \mathrm{~Hz}, 2 \mathrm{H}), 7.32-7.42(\mathrm{~m}, 5 \mathrm{H}), 4.80(\mathrm{~d}, J=4.0 \mathrm{~Hz}, 1 \mathrm{H}), 3.73(\mathrm{~s}, 3 \mathrm{H})$, $2.80(\mathrm{dd}, J=17.6,4.8 \mathrm{~Hz}, 1 \mathrm{H}), 2.62(\mathrm{~m}, 1 \mathrm{H}), 2.47(\mathrm{~m}, 1 \mathrm{H}), 2.18(\mathrm{~m}, 1 \mathrm{H}) ;{ }^{13} \mathrm{C} N M R(100.6$ $\left.\mathrm{MHz}_{\mathrm{CDCl}}\right): \delta 170.8,149.6,142.9,137.5,133.3,128.7,128.4,125.0,119.9,113.2,102.2$, 53.6, 52.8, 20.5, 18.8; MS m/z (relative intensity, \%): 320 (6.3), 319 (28.4), 260 (100), 143 (17.0), 102 (11.0), 77 (9.7); ESI-HRMS: $m / z$ Calcd for $\mathrm{C}_{19} \mathrm{H}_{17} \mathrm{~N}_{3} \mathrm{O}_{2}+\mathrm{H}^{+}$: 320.1394, found 320.1392.

methyl 6-phenyl-2-(o-tolyl)-2,3,4,5-tetrahydropyridazine-3-carboxylate (3g)<smiles>CC(=O)C1CCC(c2ccccc2)=NN1c1ccccc1C</smiles>

Yellow oil; (93 mg, 60\%); ${ }^{1} \mathrm{H}$ NMR $\left(400 \mathrm{MHz}, \mathrm{CDCl}_{3}\right): \delta 7.73(\mathrm{~d}, J=8.0 \mathrm{~Hz}, 1 \mathrm{H}), 7.29-7.34$ (m, 3H), 7.23-7.26 (m, 1H), 7.18-7.21 (m, 2H), 7.04-7.08 (m, 1H), $4.46(t, J=3.2 \mathrm{~Hz}, 1 \mathrm{H})$ $3.63(\mathrm{~s}, 3 \mathrm{H}), 2.73(\mathrm{~m}, 1 \mathrm{H}), 2.45-2.55(\mathrm{~m}, 2 \mathrm{H}), 2.31-2.40(\mathrm{~m}, 4 \mathrm{H}) ;{ }^{13} \mathrm{C} N M R(100.6 \mathrm{MHz}$, $\left.\mathrm{CDCl}_{3}\right): \delta 171.7,147.2,139.5,138.4,131.7,131.2,128.1,127.5,126.3,124.8,124.5$, 124.3, 57.2, 52.1, 21.9, 18.9, 18.8; MS m/z (relative intensity, \%): 309 (6.1), 308 (30.0), 249 (100), 143 (9.0), 91 (11.8), 77 (5.8); ESI-HRMS: m/z Calcd for $\mathrm{C}_{19} \mathrm{H}_{20} \mathrm{~N}_{2} \mathrm{O}_{2}+\mathrm{Na}^{+}$: 331.1417, found 331.1404.

methyl 6-(4-methoxyphenyl)-2-phenyl-2,3,4,5-tetrahydropyridazine-3-carboxylate (3j) 
<smiles>COc1ccc(C2=NN(c3ccccc3)C(C(C)=O)CC2)cc1</smiles>

Yellow solid; (138 mg, 85\%); mp: 95-97 ${ }^{\circ} \mathrm{C} ;{ }^{1} \mathrm{H}$ NMR (400 MHz, $\left.\mathrm{CDCl}_{3}\right): \delta 7.75(\mathrm{~m}, 2 \mathrm{H})$, 7.25-7.33 (m, 4H), 6.88-6.92 (m, 3H), $4.76(\mathrm{~d}, J=4.4 \mathrm{~Hz}, 1 \mathrm{H}), 3.83(\mathrm{~s}, 3 \mathrm{H}), 3.70(\mathrm{~s}, 3 \mathrm{H})$, $2.72(\mathrm{dd}, J=17.2,5.6 \mathrm{~Hz}, 1 \mathrm{H}), 2.55(\mathrm{~m}, 1 \mathrm{H}), 2.42(\mathrm{~m}, 1 \mathrm{H}), 2.17(\mathrm{~m}, 1 \mathrm{H}) ;{ }^{13} \mathrm{C}$ NMR $(100.6$ $\left.\mathrm{MHz}, \mathrm{CDCl}_{3}\right): \delta 172.1,159.5,146.8,139.3,131.2,129.0,126.0,120.0,113.6,113.3,55.3$ 53.8, 52.5, 20.8, 18.6; MS m/z (relative intensity, \%): 325 (11.9), 324 (60.0), 265 (100), 173 (7.2), 122 (49.3), 77 (17.0); ESI-HRMS: $m / z$ Calcd for $\mathrm{C}_{19} \mathrm{H}_{20} \mathrm{~N}_{2} \mathrm{O}_{3}+\mathrm{H}^{+}$: 325.1547, found 325.1537.

methyl 6-(4-nitrophenyl)-2-phenyl-2,3,4,5-tetrahydropyridazine-3-carboxylate (3k)<smiles>CC(=O)C1CCC(c2ccc([N+](=O)[O-])cc2)=NN1c1ccccc1</smiles>

Yellow solid; (111 mg, 65\%); $\mathrm{mp}: 147-149{ }^{\circ} \mathrm{C} ;{ }^{1} \mathrm{H}$ NMR $\left(400 \mathrm{MHz}, \mathrm{CDCl}_{3}\right): \delta 8.21(\mathrm{~d}, \mathrm{~J}=8.8$ $\mathrm{Hz}, 2 \mathrm{H}), 7.92(\mathrm{~d}, J=8.8 \mathrm{~Hz}, 2 \mathrm{H}), 7.30-7.37(\mathrm{~m}, 4 \mathrm{H}), 7.00(\mathrm{t}, J=6.4 \mathrm{~Hz}, 1 \mathrm{H}), 4.83(\mathrm{~s}, 1 \mathrm{H})$, $3.74(\mathrm{~s}, 3 \mathrm{H}), 2.76(\mathrm{dd}, J=17.2,4.0 \mathrm{~Hz}, 1 \mathrm{H}), 2.61(\mathrm{~m}, 1 \mathrm{H}), 2.49(\mathrm{~m}, 1 \mathrm{H}), 2.19(\mathrm{~m}, 1 \mathrm{H}) ;{ }^{13} \mathrm{C}$ $\operatorname{NMR}\left(100.6 \mathrm{MHz}, \mathrm{CDCl}_{3}\right): \delta 171.3,146.8,146.2,144.3,136.7,129.2,124.9,123.7,121.5$, 114.2, 54.2, 52.8, 20.4, 18.6; MS m/z (relative intensity, \%): 339 (28.4), 280 (100), 234 (30.8), 104 (6.7), 77 (17.2), 44 (20.0); ESI-HRMS: $m / z$ Calcd for $\mathrm{C}_{18} \mathrm{H}_{17} \mathrm{~N}_{3} \mathrm{O}_{4}+\mathrm{H}^{+}: 340.1292$, found 340.1285 .

methyl 6-(2-bromophenyl)-2-phenyl-2,3,4,5-tetrahydropyridazine-3-carboxylate (3I)<smiles>CC(=O)C1CCC(c2ccccc2Br)=NN1c1ccccc1</smiles>

Yellow oil; (131 mg, 70\%); ${ }^{1} \mathrm{H}$ NMR (400 MHz, $\left.\mathrm{CDCl}_{3}\right): \delta 7.60(\mathrm{~d}, J=1.2 \mathrm{~Hz}, 1 \mathrm{H}), 7.58(\mathrm{~d}, J$ 
= 1.2 Hz, 1H), $7.43(\mathrm{~m}, 1 \mathrm{H}), 7.17-7.35(\mathrm{~m}, 6 \mathrm{H}), 6.90(\mathrm{~m}, 1 \mathrm{H}), 4.77(\mathrm{dd}, J=5.2,2.0 \mathrm{~Hz}, 1 \mathrm{H})$, $3.77(\mathrm{~s}, 3 \mathrm{H}), 2.58-2.62(\mathrm{~m}, 2 \mathrm{H}), 2.50(\mathrm{~m}, 1 \mathrm{H}), 2.24(\mathrm{~m}, 1 \mathrm{H}) ;{ }^{13} \mathrm{C} \mathrm{NMR}\left(100.6 \mathrm{MHz}, \mathrm{CDCl}_{3}\right)$ : $\delta 172.2,146.6,142.3,140.8,133.2,130.7,129.2,129.0,127.3,121.7,120.4,113.6,54.3$, 52.6, 22.5, 20.8; MS m/z (relative intensity, \%): 374 (34.9), 372 (36.6), 315 (97.4), 313 (100), 233 (25.1), 104 (16.4), 77 (38.4); ESI-HRMS: m/z Calcd for $\mathrm{C}_{18} \mathrm{H}_{17} \mathrm{BrN}_{2} \mathrm{O}_{2}+\mathrm{H}^{+}$: 373.0546, found 373.0539 .

methyl 6-([1,1'-biphenyl]-4-yl)-2-phenyl-2,3,4,5-tetrahydropyridazine-3-carboxylate $(3 m)$<smiles>CC(=O)C1CCC(c2ccc(-c3ccccc3)cc2)=NN1c1ccccc1</smiles>

White solid; (93 mg, 50\%); mp: 201-203 ${ }^{\circ} \mathrm{C} ;{ }^{1} \mathrm{H}$ NMR (400 MHz, $\mathrm{CDCl}_{3}$ ): $\delta 7.89$ (d, $J=8.8$ $\mathrm{Hz}, 2 \mathrm{H}), 7.62-7.64(\mathrm{~m}, 4 \mathrm{H}), 7.45(\mathrm{t}, J=7.6 \mathrm{~Hz}, 2 \mathrm{H}), 7.32-7.37(\mathrm{~m}, 5 \mathrm{H}), 6.95(\mathrm{~m}, 1 \mathrm{H}), 4.80$ (d, $J=4.4 \mathrm{~Hz}, 1 \mathrm{H}$ ) $3.72(\mathrm{~s}, 3 \mathrm{H}), 2.80$ (dd, $J=17.2,5.2 \mathrm{~Hz}, 1 \mathrm{H}), 2.59$ (dd, $J=13.6,6.8 \mathrm{~Hz}$, 1H), $2.48(\mathrm{~m}, 1 \mathrm{H}), 2.22(\mathrm{~m}, 1 \mathrm{H}) ;{ }^{13} \mathrm{C} \mathrm{NMR}\left(100.6 \mathrm{MHz}, \mathrm{CDCl}_{3}\right): \delta 172.2,146.6,142.3$, $140.8,133.2,130.7,129.2,129.0,127.3,121.7,120.4,113.6,54.3,52.6,22.5,20.8$; MS m/z (relative intensity, \%): 371 (12.0), 370 (58.4), 311 (100), 219 (8.1), 168 (36.8), 85 (37.4), 57 (47.2); ESI-HRMS: $m / z$ Calcd for $\mathrm{C}_{24} \mathrm{H}_{22} \mathrm{~N}_{2} \mathrm{O}_{2}+\mathrm{H}^{+}: 371.1754$, found 371.1743.

methyl 2-phenyl-6-(thiophen-2-yl)-2,3,4,5-tetrahydropyridazine-3-carboxylate (3n)<smiles>CC(=O)C1CCC(c2cccs2)=NN1c1ccccc1</smiles>

Brown solid; (105 mg, 70\%); mp: $128-130{ }^{\circ} \mathrm{C} ;{ }^{1} \mathrm{H}$ NMR (400 MHz, $\mathrm{CDCl}_{3}$ ): $\delta 7.29-7.33(\mathrm{~m}$, 2H), 7.21-7.24 (m, 3H), $7.09(\mathrm{~d}, J=3.2 \mathrm{~Hz}, 1 \mathrm{H}), 6.98(\mathrm{dd}, J=5.2,4.0 \mathrm{~Hz}, 1 \mathrm{H}), 6.91(\mathrm{t}, J$ $=7.2 \mathrm{~Hz}, 1 \mathrm{H}), 4.77(\mathrm{~d}, J=4.8 \mathrm{~Hz}, 1 \mathrm{H}) 3.71(\mathrm{~s}, 3 \mathrm{H}), 2.70-2.75(\mathrm{~m}, 1 \mathrm{H}), 2.41-2.55(\mathrm{~m}, 2 \mathrm{H})$, 2.13-2.22 (m, 1H); ${ }^{13} \mathrm{C}$ NMR $\left(100.6 \mathrm{MHz}, \mathrm{CDCl}_{3}\right): \delta 171.8,146.3,144.5,136.7,129.1$, $126.9,125.5,122.9,120.4,113.4,54.1,52.6,20.6,19.3 ; \mathrm{MS} \mathrm{m} / \mathrm{z}$ (relative intensity, \%): 301 (9.1), 300 (50.2), 241 (100), 208 (4.9), 144 (14.1), 98 (14.7), 77 (15.6); ESI-HRMS: m/z 
Calcd for $\mathrm{C}_{16} \mathrm{H}_{16} \mathrm{~N}_{2} \mathrm{O}_{2} \mathrm{~S}+\mathrm{H}^{+}$: 301.1005, found 301.1001 .

methyl 6-methyl-2-phenyl-2,3,4,5-tetrahydropyridazine-3-carboxylate (3o)<smiles>CC(=O)C1CCC(C)=NN1c1ccccc1</smiles>

Yellow solid; (48 mg, 41\%); mp: $107-109{ }^{\circ} \mathrm{C} ;{ }^{1} \mathrm{H}$ NMR (400 MHz, $\left.\mathrm{CDCl}_{3}\right): \delta 7.25$ (t, J=8.0 $\mathrm{Hz}, 2 \mathrm{H}), 7.14(\mathrm{~d}, J=8.0 \mathrm{~Hz}, 2 \mathrm{H}), 6.84(\mathrm{t}, J=7.2 \mathrm{~Hz}, 1 \mathrm{H}), 4.64(\mathrm{~s}, 1 \mathrm{H}), 3.70(\mathrm{~s}, 3 \mathrm{H}), 2.38(\mathrm{~m}$, 1H), $2.11(\mathrm{~m}, 3 \mathrm{H}), 2.01(\mathrm{~s}, 3 \mathrm{H}) ;{ }^{13} \mathrm{C} \mathrm{NMR}\left(100.6 \mathrm{MHz}, \mathrm{CDCl}_{3}\right): \delta 172.3,147.0,142.3,129.0$, 119.4, 112.9, 53.3, 52.4, 24.2, 22.5, 20.9; MS m/z (relative intensity, \%): 232 (29.5), 173 (100), 158 (12.4), 104 (9.6), 77 (22.1); ESI-HRMS: $m / z$ Calcd for $\mathrm{C}_{13} \mathrm{H}_{16} \mathrm{~N}_{2} \mathrm{O}_{2}+\mathrm{H}^{+}: 233.1285$, found 233.1281 .

methyl 6-(tert-butyl)-2-phenyl-2,3,4,5-tetrahydropyridazine-3-carboxylate (3p)<smiles>CC(=O)C1CCC(C(C)(C)C)=NN1c1ccccc1</smiles>

Colorless oil; (75.5 mg, 55\%); ${ }^{1} \mathrm{H}$ NMR (400 MHz, $\left.\mathrm{CDCl}_{3}\right): \delta 7.24(\mathrm{dd}, J=8.8,1.2 \mathrm{~Hz}, 2 \mathrm{H})$, $7.15(\mathrm{~d}, J=8.0 \mathrm{~Hz}, 2 \mathrm{H}), 6.82(\mathrm{t}, J=7.2 \mathrm{~Hz}, 1 \mathrm{H}), 4.61(\mathrm{~d}, J=3.2 \mathrm{~Hz}, 1 \mathrm{H}), 3.69(\mathrm{~s}, 3 \mathrm{H})$, 2.28-2.39 (m, 2H), 1.95-2.07 (m, 2H), $1.82(\mathrm{~s}, 9 \mathrm{H}) ;{ }^{13} \mathrm{C} \mathrm{NMR}\left(100.6 \mathrm{MHz}, \mathrm{CDCl}_{3}\right): \delta 172.5$, $150.4,147.1,128.9,119.1,112.5,53.6,52.3,38.2,27.8,21.1,16.8 ; \mathrm{MS} m / z$ (relative intensity, \%): 274 (32.4), 215 (100), 159 (26.8), 77 (11.4), 57 (24.3); ESI-HRMS: m/z Calcd for $\mathrm{C}_{16} \mathrm{H}_{22} \mathrm{~N}_{2} \mathrm{O}_{2}+\mathrm{H}^{+}: 275.1754$, found 275.1760 .

ethyl 2,6-diphenyl-2,3,4,5-tetrahydropyridazine-3-carboxylate (3r)<smiles>CCOC(=O)C1CCC(c2ccccc2)=NN1c1ccccc1</smiles>

Yellow oil; (128 mg, 83\%); ${ }^{1} \mathrm{H}$ NMR (400 MHz, $\left.\mathrm{CDCl}_{3}\right): \delta 7.81(\mathrm{~d}, J=7.6 \mathrm{~Hz}, 2 \mathrm{H}), 7.36(\mathrm{~m}$, $2 \mathrm{H}), 7.24-7.31(\mathrm{~m}, 5 \mathrm{H}), 6.92(\mathrm{~m}, 1 \mathrm{H}), 4.75(\mathrm{~d}, J=4.8 \mathrm{~Hz}, 1 \mathrm{H}), 4.18(\mathrm{q}, J=7.2 \mathrm{~Hz}, 2 \mathrm{H}), 2.75$ 
(dd, $J=16.8,4.8 \mathrm{~Hz}, 1 \mathrm{H}), 2.55(\mathrm{~m}, 1 \mathrm{H}), 2.46(\mathrm{~m}, 1 \mathrm{H}), 2.17(\mathrm{~m}, 1 \mathrm{H}), 1.19(\mathrm{t}, J=7.2 \mathrm{~Hz}, 3 \mathrm{H})$; ${ }^{13} \mathrm{C}$ NMR $\left(100.6 \mathrm{MHz}, \mathrm{CDCl}_{3}\right): \delta 171.4,146.8,139.2,138.4,129.0,128.2,127.8,124.6$, 120.2, 113.6, 61.4, 54.1, 20.7, 18.6, 14.2; MS m/z (relative intensity, \%): 308 (33.7), 249 (24.8), 235 (100), 143 (13.6), 104 (8.0), 77 (23.4); ESI-HRMS: m/z Calcd for $\mathrm{C}_{19} \mathrm{H}_{20} \mathrm{~N}_{2} \mathrm{O}_{2}+\mathrm{H}^{+}:$309.1598, found 309.1586.

butyl 2,6-diphenyl-2,3,4,5-tetrahydropyridazine-3-carboxylate (3s)<smiles>CCOC(=O)C1CCC(c2ccccc2)=NN1c1ccccc1</smiles>

Yellow oil; (146 mg, 88\%); $\left.{ }^{1} \mathrm{H} \mathrm{NMR} \mathrm{(400} \mathrm{MHz,} \mathrm{CDCl}_{3}\right): \delta 7.81$ (d, $\left.J=8.0 \mathrm{~Hz}, 2 \mathrm{H}\right), 7.36$ (t, $J=$ $7.6 \mathrm{~Hz}, 2 \mathrm{H}), 7.25-7.31(\mathrm{~m}, 5 \mathrm{H}), 6.92(\mathrm{~m}, 1 \mathrm{H}), 4.77(\mathrm{~d}, J=4.4 \mathrm{~Hz}, 1 \mathrm{H}), 4.12(\mathrm{t}, J=6.8 \mathrm{~Hz}$, $2 \mathrm{H}), 2.75(\mathrm{dd}, J=16.8,4.8 \mathrm{~Hz}, 1 \mathrm{H}), 2.56(\mathrm{~m}, 1 \mathrm{H}), 2.46(\mathrm{~m}, 1 \mathrm{H}), 2.19(\mathrm{~m}, 1 \mathrm{H}), 1.53(\mathrm{~m}, 2 \mathrm{H})$, $1.24(\mathrm{~m}, 2 \mathrm{H}), 0.82(\mathrm{t}, J=7.6 \mathrm{~Hz}, 3 \mathrm{H}) ;{ }^{13} \mathrm{C} \mathrm{NMR}\left(100.6 \mathrm{MHz}, \mathrm{CDCl}_{3}\right): \delta 171.4,146.7,139.1$, $138.3,128.8,128.0,127.6,124.5,120.0,113.5,65.1$, 54.0, 30.3, 20.7, 18.7, 18.6, 13.3; MS m/z (relative intensity, \%): 336 (18.0), 280 (17.7), 236 (16.5), 235 (100), 143 (8.0), 77 (13.2); ESI-HRMS: $m / z$ Calcd for $\mathrm{C}_{21} \mathrm{H}_{24} \mathrm{~N}_{2} \mathrm{O}_{2}+\mathrm{H}^{+}$: 337.1911, found 337.1901.

tert-butyl 2,6-diphenyl-2,3,4,5-tetrahydropyridazine-3-carboxylate (3t)<smiles>CCCOC(=O)C1CCC(c2ccccc2)=NN1c1ccccc1</smiles>

Yellow oil; (143 mg, 85\%); ${ }^{1} \mathrm{H}$ NMR (400 MHz, CDCl 3 ): $\delta 7.80(\mathrm{~d}, J=7.6 \mathrm{~Hz}, 2 \mathrm{H}), 7.36(\mathrm{~m}$, $2 \mathrm{H}), 7.23-7.30(\mathrm{~m}, 5 \mathrm{H}), 6.91(\mathrm{~m}, 1 \mathrm{H}), 4.63(\mathrm{~d}, J=4.4 \mathrm{~Hz}, 1 \mathrm{H}), 2.74(\mathrm{~m}, 1 \mathrm{H}), 2.51(\mathrm{~m}, 1 \mathrm{H})$, $2.45(\mathrm{~m}, 1 \mathrm{H}), 2.14(\mathrm{~m}, 1 \mathrm{H}), 1.38(\mathrm{~s}, 9 \mathrm{H}) ;{ }^{13} \mathrm{C} \mathrm{NMR}\left(100.6 \mathrm{MHz}, \mathrm{CDCl}_{3}\right): \delta 170.4,147.0$, $139.1,138.6,128.9,128.2,127.6,124.7,120.0,113.6,82.0,54.7,27.9,20.8,18.6$; MS m/z (relative intensity, \%): 336 (20.5), 280 (24.8), 236 (16.4), 235 (100), 143 (8.3), 77 (13.6); ESI-HRMS: $m / z$ Calcd for $\mathrm{C}_{21} \mathrm{H}_{24} \mathrm{~N}_{2} \mathrm{O}_{2}+\mathrm{H}^{+}$: 337.1911, found 373.1903.

benzyl 2,6-diphenyl-2,3,4,5-tetrahydropyridazine-3-carboxylate (3u) 
<smiles>O=C(Cc1ccccc1)C1CCC(c2ccccc2)=NN1c1ccccc1</smiles>

Pale yellow solid; (141 mg, 76\%); mp: $109-111{ }^{\circ} \mathrm{C} ;{ }^{1} \mathrm{H}$ NMR (400 MHz, $\mathrm{CDCl}_{3}$ ): $\delta 7.76$ (d, J $=7.2 \mathrm{~Hz}, 2 \mathrm{H}), 7.19-7.37(\mathrm{~m}, 12 \mathrm{H}), 6.91(\mathrm{~s}, 1 \mathrm{H}), 5.12(\mathrm{~m}, 2 \mathrm{H}), 4.78(\mathrm{~d}, J=2.0 \mathrm{~Hz}, 1 \mathrm{H}), 2.64$ $(\mathrm{dd}, J=17.2,4.8 \mathrm{~Hz}, 1 \mathrm{H}), 2.50(\mathrm{~m}, 1 \mathrm{H}), 2.31(\mathrm{~m}, 1 \mathrm{H}), 2.12(\mathrm{~m}, 1 \mathrm{H}) ;{ }^{13} \mathrm{C}$ NMR $(100.6 \mathrm{MHz}$, $\left.\mathrm{CDCl}_{3}\right): \delta 171.1,146.8,139.5,138.3,135.4,129.0,128.4,128.14,128.06,127.9,127.7$, 124.6, 120.2, 113.7, 66.9, 54.0, 20.8, 18.6; MS m/z (relative intensity, \%): 370 (25.4), 294 (5.3), 249 (16.1), 235 (100), 179 (11.8), 143 (12.5), 107 (14.7), 77 (25.9); ESI-HRMS: m/z Calcd for $\mathrm{C}_{24} \mathrm{H}_{22} \mathrm{~N}_{2} \mathrm{O}_{2}+\mathrm{H}^{+}: 371.1754$, found 371.1749 .

\section{phenyl 2,6-diphenyl-2,3,4,5-tetrahydropyridazine-3-carboxylate (3v)}<smiles>O=C(c1ccccc1)C1CCC(c2ccccc2)=NN1c1ccccc1</smiles>

Pale yellow solid; (121 mg, 68\%); mp: $110-112{ }^{\circ} \mathrm{C} ;{ }^{1} \mathrm{H}$ NMR (400 MHz, $\left.\mathrm{CDCl}_{3}\right): \delta 7.75$ (d, J = 7.6 Hz, 2H), 7.21-7.34 (m, 9H), $7.13(\mathrm{~m}, 1 \mathrm{H}), 6.86-6.92(\mathrm{~m}, 3 \mathrm{H}), 4.91(\mathrm{~d}, J=4.0 \mathrm{~Hz}, 1 \mathrm{H})$, $2.77(\mathrm{dd}, J=17.2,5.2 \mathrm{~Hz}, 1 \mathrm{H}), 2.64(\mathrm{~m}, 1 \mathrm{H}), 2.53(\mathrm{~m}, 1 \mathrm{H}), 2.24(\mathrm{~m}, 1 \mathrm{H}) ;{ }^{13} \mathrm{C} N M R(100.6$ $\mathrm{MHz}_{\mathrm{CDCl}}$ ): $\delta$ 170.0, 150.4, 146.8, 139.6, 138.3, 129.4, 129.1, 128.3, 128.0, 126.1, 124.8, 121.2, 120.6, 113.8, 54.2, 21.0, 18.7; MS m/z (relative intensity, \%): 357 (7.9), 356 (32.2), 303 (8.7), 236 (16.3), 235 (100), 143 (12.3), 77 (17.4); ESI-HRMS: m/z Calcd for $\mathrm{C}_{23} \mathrm{H}_{20} \mathrm{~N}_{2} \mathrm{O}_{2}+\mathrm{H}^{+}:$357.1598, found 357.1590.

\section{N,2,6-triphenyl-2,3,4,5-tetrahydropyridazine-3-carboxamide (3w)}<smiles>NC(=O)C1CCC(c2ccccc2)=NN1c1ccccc1</smiles>

White solid; (98 mg, 55\%); mp: $215-216{ }^{\circ} \mathrm{C} ;{ }^{1} \mathrm{H}$ NMR (400 MHz, DMSO-d ) $^{\circ} \delta 10.34$ (brs, $\mathrm{NH}, 1 \mathrm{H}), 7.81(\mathrm{~d}, J=7.6 \mathrm{~Hz}, 2 \mathrm{H}), 7.59(\mathrm{~d}, J=8.0 \mathrm{~Hz}, 2 \mathrm{H}), 7.41(\mathrm{t}, J=7.6 \mathrm{~Hz}, 2 \mathrm{H}), 7.29-7.33$ (m, 7H), $7.06(t, J=7.2,7.2 \mathrm{~Hz}, 1 \mathrm{H}), 6.85(\mathrm{t}, J=6.0 \mathrm{~Hz}, 1 \mathrm{H}), 4.92(\mathrm{~d}, J=4.0 \mathrm{~Hz}, 1 \mathrm{H}), 2.76$ $(\mathrm{dd}, J=17.6,5.2 \mathrm{~Hz}, 1 \mathrm{H}), 2.37-2.50(\mathrm{~m}, 2 \mathrm{H}), 2.23(\mathrm{~m}, 1 \mathrm{H}) ;{ }^{13} \mathrm{C} N M R(100.6 \mathrm{MHz}$, 
DMSO- $\left.d_{6}\right): \delta 169.4,146.7,138.9,138.7,138.3,128.9,128.7,128.2,127.5,124.3,123.5$, 119.4, 119.3, 112.8, 54.5, 21.1, 18.0; MS m/z (relative intensity, \%): 356 (3.5), 355 (11.5), 235 (100), 143 (9.8), 104 (5.2), 77 (12.3); ESI-HRMS: m/z Calcd for $\mathrm{C}_{23} \mathrm{H}_{21} \mathrm{~N}_{3} \mathrm{O}+\mathrm{H}^{+}$: 356.1757 , found 356.1754 .

$\left(1 S^{*}, 2 S^{*}, 4 S^{*}\right)-1,7,7-t r i m e t h y l b i c y c l o[2.2 .1]$ heptan-2-yl-2,6-diphenyl-2,3,4,5-tetrahydro pyridazine-3-carboxylate (3x)<smiles>CC1(C)[C@H]2CC[C@@]1(C)C(OC(=O)C1CCC(c3ccccc3)=NN1c1ccccc1)C2</smiles>

Colorless oil; (156 mg, 75\%); ${ }^{1} \mathrm{H}$ NMR (400 MHz, $\mathrm{CDCl}_{3}$ ): $\delta$ 7.77-7.81 (m, 5.4H), 7.27-7.39 (m, 18.9H), 6.88-6.92 (m, 2.7H), 4.76-4.77 (m, 2.7H), 4.61-4.66 (m, 2.7H), 2.73-2.78 (m, 2.7H), 2.55-2.62 (m, 2.7H), 2.36-2.47 (m, 2.7H), 2.14-2.22 (m, 2.7H), 1.68-1.78 (m, 2.7H), 1.55-1.62 (m, 5.4H), 1.43-1.48 $(\mathrm{m}, 2.7 \mathrm{H}), 0.94-1.07(\mathrm{~m}, 6.4 \mathrm{H}), 0.82-0.84(\mathrm{~m}$, 2.7H), $0.67(\mathrm{~s}, 5 \mathrm{H}), 0.66(\mathrm{~s}, 3 \mathrm{H}), 0.64(\mathrm{~s}, 3 \mathrm{H}), 0.62(\mathrm{~s}, 5 \mathrm{H}), 0.51(\mathrm{~s}, 3 \mathrm{H}), 0.48(\mathrm{~s}, 5 \mathrm{H}) ;{ }^{13} \mathrm{C}$ NMR $\left(100.6 \mathrm{MHz}, \mathrm{CDCl}_{3}\right): \delta 171.4,171.3,146.74,146.72,139.02,138.95,138.5,138.4$, 128.91, 128.89, 128.17, 128.16, 127.7, 124.62, 124.60, 119.97, 119.95, 113.2, 113.1, $82.6,82.4,53.84,53.78,48.6,48.3,46.8,44.80,44.76,39.0,38.7,33.53,33.51,26.8$, 21.1, 20.8, 19.9, 19.8, 19.5, 19.2; MS m/z (relative intensity, \%): 416 (13.9), 280 (32.2), 235 (100), 143 (7.8), 137 (4.9), 77 (8.8); ESI-HRMS: m/z Calcd for $\mathrm{C}_{27} \mathrm{H}_{32} \mathrm{~N}_{2} \mathrm{O}_{2}+\mathrm{H}^{+}$: 417.2537, found 417.2545 .

\section{1,3,6-triphenyl-1,4,5,6-tetrahydropyridazine (3y)}<smiles>Pc1ccccc1</smiles>

Pale yellow solid; (86 mg, 55\%); mp: $132-133{ }^{\circ} \mathrm{C} ;{ }^{1} \mathrm{H}$ NMR (400 MHz, CDCl 3 ): $\delta 7.82$ (d, J $=7.6 \mathrm{~Hz}, 2 \mathrm{H}), 7.38(\mathrm{t}, J=7.6 \mathrm{~Hz}, 2 \mathrm{H}), 7.21-7.30(\mathrm{~m}, 8 \mathrm{H}), 7.17(\mathrm{~d}, J=7.2 \mathrm{~Hz}, 2 \mathrm{H}), 6.84(\mathrm{t}, J$ $=6.8 \mathrm{~Hz}, 1 \mathrm{H}), 5.26(\mathrm{~s}, 1 \mathrm{H}), 2.63(\mathrm{~d}, J=13.2 \mathrm{~Hz}, 1 \mathrm{H}), 2.31(\mathrm{~m}, 2 \mathrm{H}), 2.14(\mathrm{~m}, 1 \mathrm{H}) ;{ }^{13} \mathrm{C} \mathrm{NMR}$ $\left(100.6 \mathrm{MHz}_{\mathrm{CDCl}}\right)$ : $\delta$ 146.7, 140.9, 139.2, 138.8, 128.9, 128.8, 128.3, 127.5, 127.1, 126.0, 
124.5, 119.6, 113.7, 55.4, 25.0, 17.6; MS m/z (relative intensity, \%): 313 (24.3), 312 (100), 235 (29.8), 103 (44.3), 92 (16.7), 77 (50.1); ESI-HRMS: $m / z$ Calcd for $\mathrm{C}_{22} \mathrm{H}_{20} \mathrm{~N}_{2}+\mathrm{H}^{+}$: 313.1699, found 313.1690.

\section{1,3-diphenyl-6-(p-tolyl)-1,4,5,6-tetrahydropyridazine (3z)}<smiles>Cc1ccc(C2CCC(c3ccccc3)=NN2c2ccccc2)cc1</smiles>

Pale oil; (73 mg, 45\%); $\left.{ }^{1} \mathrm{H} \mathrm{NMR} \mathrm{(400} \mathrm{MHz,} \mathrm{CDCl}_{3}\right): 7.83$ (d, $\left.J=8.0 \mathrm{~Hz}, 2 \mathrm{H}\right), 7.38$ (t, $J=7.6$ $\mathrm{Hz}, 2 \mathrm{H}), 7.21-7.31(\mathrm{~m}, 5 \mathrm{H}), 7.04-7.10(\mathrm{~m}, 4 \mathrm{H}), 6.84(\mathrm{t}, J=7.2 \mathrm{~Hz}, 1 \mathrm{H}), 5.23(\mathrm{~s}, 1 \mathrm{H}), 2.61$ $(\mathrm{d}, J=16.8 \mathrm{~Hz}, 1 \mathrm{H}), 2.27-2.32(\mathrm{~m}, 5 \mathrm{H}), 2.15(\mathrm{~m}, 1 \mathrm{H}) ;{ }^{13} \mathrm{C} \mathrm{NMR}\left(100.6 \mathrm{MHz}, \mathrm{CDCl}_{3}\right): \delta$ $146.7,139.1,138.8,137.9,136.7,129.4,128.9,128.3,127.5,125.9,124.5,119.6,113.6$, 55.2, 25.1, 21.0, 17.6; MS m/z (relative intensity, \%): 326 (100), 233 (29.7), 207 (21.6), 156 (22.7), 103 (25.2), 77 (30.0); ESI-HRMS: $m / z$ Calcd for $\mathrm{C}_{23} \mathrm{H}_{22} \mathrm{~N}_{2}+\mathrm{H}^{+}: 327.1856$, found 327.1849 .

6-(4-nitrophenyl)-1,3-diphenyl-1,4,5,6-tetrahydropyridazine (3aa)<smiles>O=[N+]([O-])c1ccc(C2CCC(c3ccccc3)=NN2c2ccccc2)cc1</smiles>

Yellow solid; (84 mg, 47\%); mp: $138-139{ }^{\circ} \mathrm{C} ;{ }^{1} \mathrm{H}$ NMR (400 MHz, $\mathrm{CDCl}_{3}$ ): $\delta 8.16$ (d, $\mathrm{J}=8.4$ $\mathrm{Hz}, 2 \mathrm{H}), 7.82(\mathrm{~d}, J=7.6 \mathrm{~Hz}, 2 \mathrm{H}), 7.30-7.42(\mathrm{~m}, 5 \mathrm{H}), 7.24-7.25(\mathrm{~m}, 4 \mathrm{H}), 6.88(\mathrm{~s}, 1 \mathrm{H}), 5.36$ (s, $1 \mathrm{H}), 2.69(\mathrm{~d}, J=18.0 \mathrm{~Hz}, 1 \mathrm{H}), 2.36-2.44(\mathrm{~m}, 2 \mathrm{H}), 2.07(\mathrm{~m}, 1 \mathrm{H}) ;{ }^{13} \mathrm{C} \mathrm{NMR}(100.6 \mathrm{MHz}$, $\left.\mathrm{CDCl}_{3}\right): \delta 148.6,147.2,146.2,139.6,138.2,129.1,128.4,128.0,127.1,124.5,124.2$, 120.2, 113.6, 55.0, 24.7, 17.5; MS m/z (relative intensity, \%): 357 (100), 280 (6.0), 235 (24.5), 207 (14.7), 105 (12.6), 77 (39.9); ESI-HRMS: m/z Calcd for $\mathrm{C}_{22} \mathrm{H}_{19} \mathrm{~N}_{3} \mathrm{O}_{2}+\mathrm{H}^{+}$: 358.1550 , found 358.1545 .

\section{1,3-diphenyl-6-(pyridin-2-yl)-1,4,5,6-tetrahydropyridazine (3ab)}


<smiles>Pc1ccccc1</smiles>

Yellow solid; (78 mg, 50\%); $\mathrm{mp}: 129-130{ }^{\circ} \mathrm{C} ;{ }^{1} \mathrm{H}$ NMR (400 MHz, $\left.\mathrm{CDCl}_{3}\right): \delta 8.65$ (d, $J=4.0$ $\mathrm{Hz}, 1 \mathrm{H}), 7.82$ (d, J= 7.2 Hz, 2H), 7.48 (td, J=8.0, $1.6 \mathrm{~Hz}, 1 \mathrm{H}), 7.37$ (t, $J=7.6 \mathrm{~Hz}, 2 \mathrm{H}$ ), 7.22-7.32 (m, 5H), 7.13 (dd, J = 6.8, 4.8 Hz, 1H), $7.00(\mathrm{~d}, J=8.0 \mathrm{~Hz}, 1 \mathrm{H}), 6.88(\mathrm{t}, J=7.2 \mathrm{~Hz}$, $1 \mathrm{H}), 5.35(\mathrm{~d}, J=3.6 \mathrm{~Hz}, 1 \mathrm{H}), 2.32-2.72(\mathrm{~m}, 2 \mathrm{H}), 2.28(\mathrm{~m}, 1 \mathrm{H}), 2.04(\mathrm{~m}, 1 \mathrm{H}) ;{ }^{13} \mathrm{C} N M R$ $\left(100.6 \mathrm{MHz}, \mathrm{CDCl}_{3}\right): \delta 159.7,150.0,146.6,139.6,138.5,136.7,128.9,128.2,127.6$, $124.5,122.0,121.0,119.8,113.5,57.0,22.8,17.9 ; \mathrm{MS} \mathrm{m} / \mathrm{z}$ (relative intensity, \%): 313 (100), 312 (25.3), 235 (27.4), 215 (19.1), 207 (11.6), 103 (25.0), 77 (38.7); ESI-HRMS: m/z Calcd for $\mathrm{C}_{21} \mathrm{H}_{19} \mathrm{~N}_{3}+\mathrm{H}^{+}$: 314.1652, found 314.1656.

\section{1,3-diphenyl-6-(pyridin-4-yl)-1,4,5,6-tetrahydropyridazine (3ac)}<smiles>c1ccc(C2=NN(c3ccccc3)C(c3ccncc3)CC2)cc1</smiles>

Pale yellow solid; (80 mg, 50\%); mp: $127-128{ }^{\circ} \mathrm{C} ;{ }^{1} \mathrm{H} \mathrm{NMR}\left(400 \mathrm{MHz}, \mathrm{CDCl}_{3}\right): \delta 8.53$ (d, J= $5.6 \mathrm{~Hz}, 2 \mathrm{H}), 7.81(\mathrm{~d}, J=7.2 \mathrm{~Hz}, 2 \mathrm{H}), 7.38(\mathrm{t}, J=7.6 \mathrm{~Hz}, 2 \mathrm{H}), 7.30(\mathrm{t}, J=7.2 \mathrm{~Hz}, 1 \mathrm{H})$, 7.24-7.25 (m, 4H), $7.11(\mathrm{~d}, J=6.0 \mathrm{~Hz}, 2 \mathrm{H}), 6.88(\mathrm{~m}, 1 \mathrm{H}), 5.24(\mathrm{~s}, 1 \mathrm{H}), 2.65(\mathrm{~m}, 1 \mathrm{H})$, 2.32-2.37 (m, 2H), $2.07(\mathrm{~m}, 1 \mathrm{H}) ;{ }^{13} \mathrm{C}$ NMR (100.6 MHz, $\left.\mathrm{CDCl}_{3}\right): \delta 150.3,150.0,146.4$, $139.6,138.7,129.0,128.3,127.9,124.6,121.3,120.2,113.6,54.6,24.4,17.6$; MS m/z (relative intensity, \%): 313 (100), 312 (22.3), 235 (28.2), 207 (16.4), 103 (27.6), 77 (38.1); ESI-HRMS: $m / z$ Calcd for $\mathrm{C}_{21} \mathrm{H}_{19} \mathrm{~N}_{3}+\mathrm{H}^{+}$: 314.1652, found 314.1646.

\section{methyl 3-methyl-2,6-diphenyl-2,3,4,5-tetrahydropyridazine-3-carboxylate (3ae)}<smiles>COC(=O)C1(C)CCC(c2ccccc2)=NN1c1ccccc1</smiles>

Pale yellow oil; (112 mg, 73\%); ${ }^{1} \mathrm{H}$ NMR (400 MHz, CDCl $): \delta 7.76(\mathrm{~d}, J=7.6 \mathrm{~Hz}, 2 \mathrm{H}), 7.34$ (t, $J=7.6 \mathrm{~Hz}, 2 \mathrm{H}), 7.24-7.30(\mathrm{~m}, 5 \mathrm{H}), 6.98(\mathrm{t}, J=6.8 \mathrm{~Hz}, 1 \mathrm{H}), 3.67(\mathrm{~s}, 3 \mathrm{H}), 2.63(\mathrm{~m}, 2 \mathrm{H})$, $2.40(\mathrm{~m}, 1 \mathrm{H}), 2.06(\mathrm{~m}, 1 \mathrm{H}), 1.62(\mathrm{~s}, 3 \mathrm{H}) ;{ }^{13} \mathrm{C} \mathrm{NMR}\left(100.6 \mathrm{MHz}, \mathrm{CDCl}_{3}\right): \delta 175.0,146.3$, 
$138.6,138.3,128.3,128.2,127.6,124.5,122.0,119.7,59.8 .52 .6,31.1,21.6,19.3$; MS m/z (relative intensity, \%): 308 (13.5), 249 (100), 157 (9.6), 118 (6.7), 103 (7.8), 77 (18.5); ESI-HRMS: $m / z$ Calcd for $\mathrm{C}_{19} \mathrm{H}_{20} \mathrm{~N}_{2} \mathrm{O}_{2}+\mathrm{H}^{+}: 309.1598$, found 309.1587.

\section{6-methyl-1,3,6-triphenyl-1,4,5,6-tetrahydropyridazine (3af)}<smiles>CC1(c2ccccc2)CCC(c2ccccc2)=NN1c1ccccc1</smiles>

Yellow solid; (82 mg, 50\%); mp: $133-135{ }^{\circ} \mathrm{C} ;{ }^{1} \mathrm{H} \mathrm{NMR}\left(400 \mathrm{MHz}, \mathrm{CDCl}_{3}\right): \delta 7.83(\mathrm{~d}, J=7.2$ $\mathrm{Hz}, 2 \mathrm{H}), 7.39(\mathrm{~m}, 6 \mathrm{H}), 7.30(\mathrm{~m}, 2 \mathrm{H}), 7.23(\mathrm{~m}, 4 \mathrm{H}), 6.98(\mathrm{~m}, 1 \mathrm{H}), 2.65(\mathrm{~m}, 1 \mathrm{H}), 2.29(\mathrm{~m}, 1 \mathrm{H})$, $2.18(\mathrm{~m}, 2 \mathrm{H}), 1.90(\mathrm{~s}, 3 \mathrm{H}) ;{ }^{13} \mathrm{C}$ NMR (100.6 MHz, $\left.\mathrm{CDCl}_{3}\right): \delta$ 146.4, 145.3, 138.8, 138.1, 128.6, 128.2, 127.8, 127.1, 126.8, 125.8, 124.3, 121.3, 121.2, 59.7, 36.6, 26.3, 19.9; MS m/z (relative intensity, \%): 327 (23.5), 326 (100), 280 (7.6), 235 (42.6), 219 (16.5), 207 (16.5), 103 (43.7), 77 (54.2); ESI-HRMS: $m / z$ Calcd for $\mathrm{C}_{23} \mathrm{H}_{22} \mathrm{~N}_{2}+\mathrm{H}^{+}$: 327.1856, found 327.1844 .

\section{ORTEP Plot for Compound 3y:}

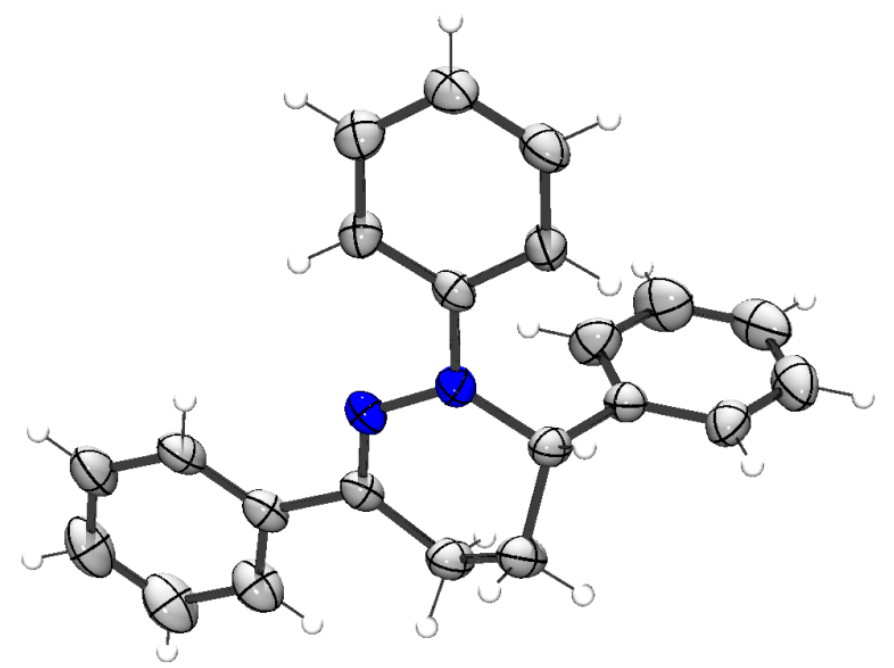

Figure S-1. ORTEP of compound $\mathbf{3 y}$, thermal ellipsoids are drawn at $30 \%$ probability level. 


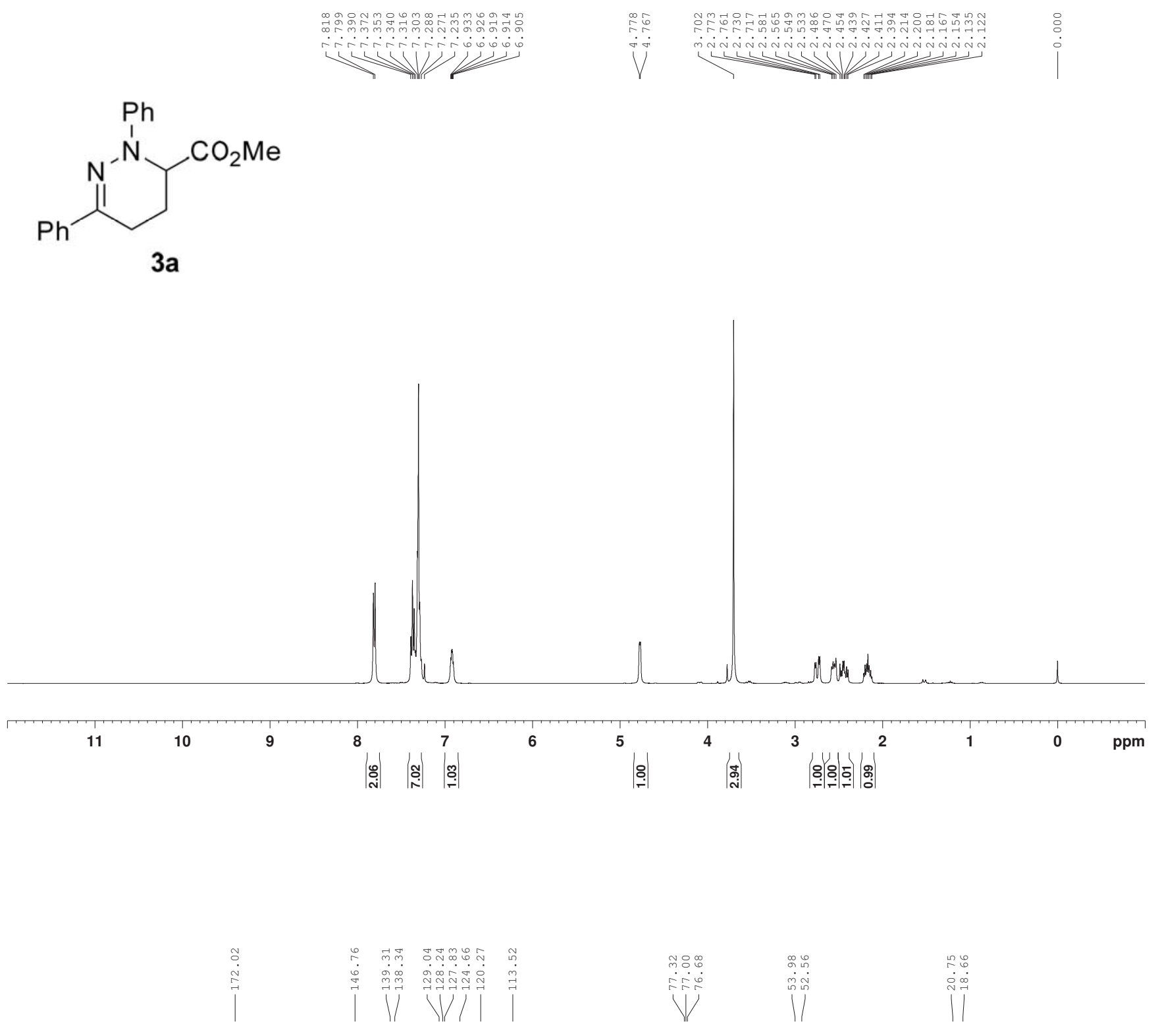<smiles>COC(=O)C1CCC(c2ccccc2)=NN1c1ccccc1</smiles>

$3 a$ 

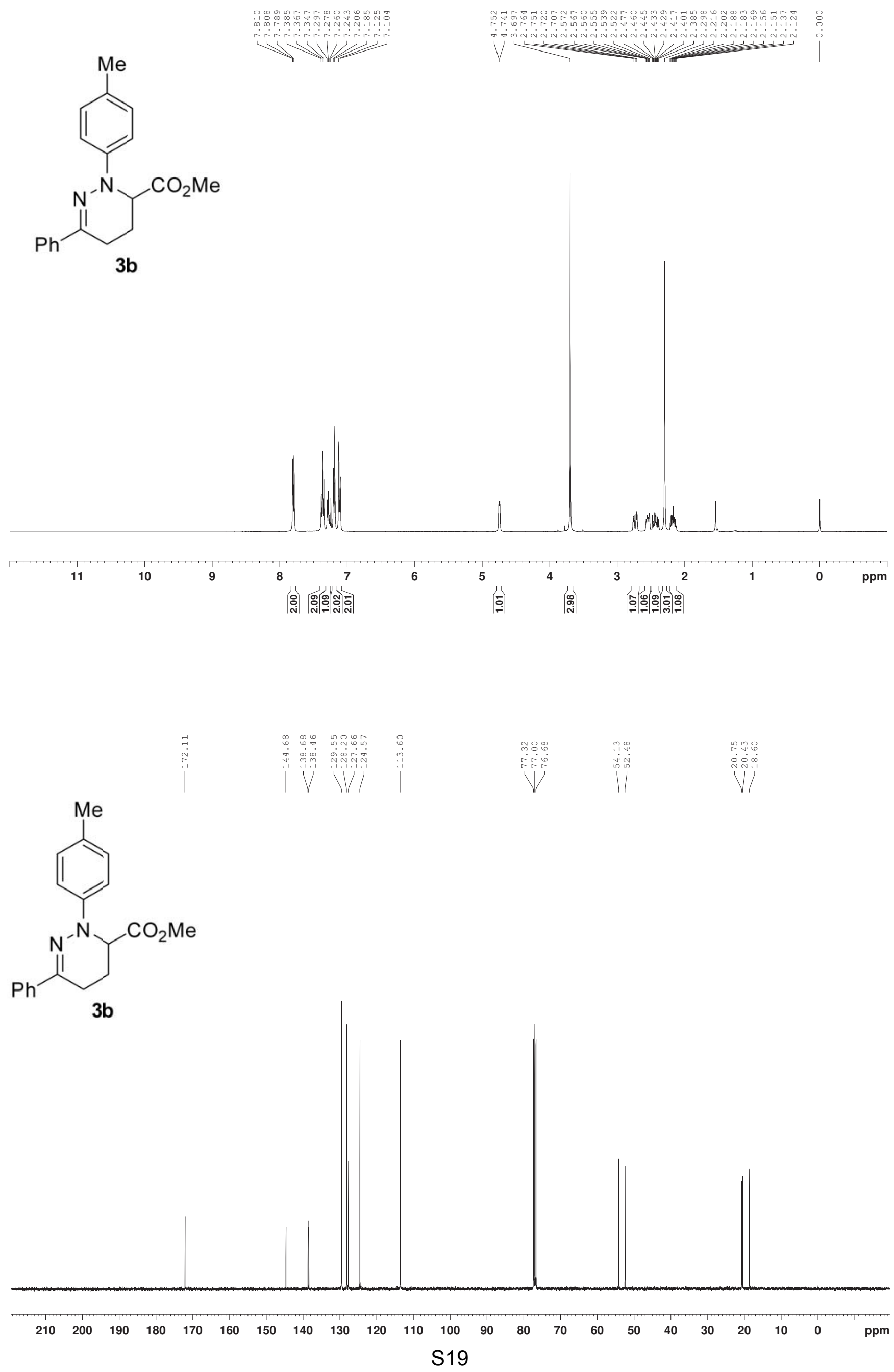

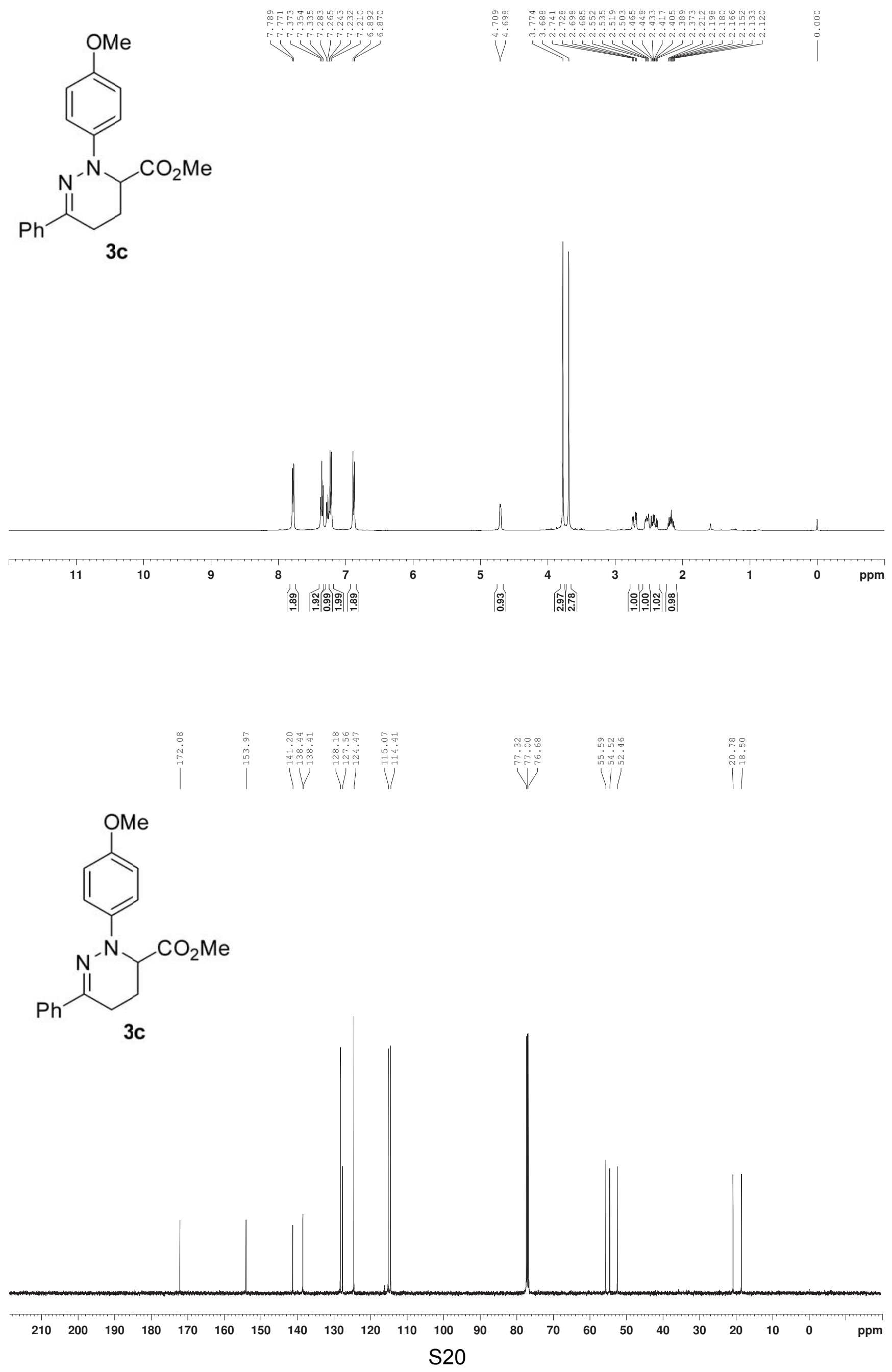
<smiles>COC(=O)C1CCC(c2ccccc2)=NN1c1ccc(Cl)cc1</smiles>

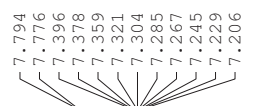

$3 d$

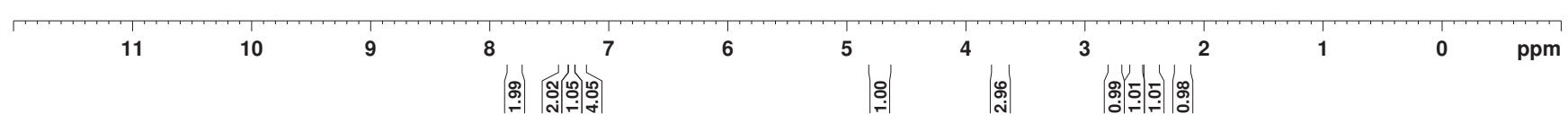
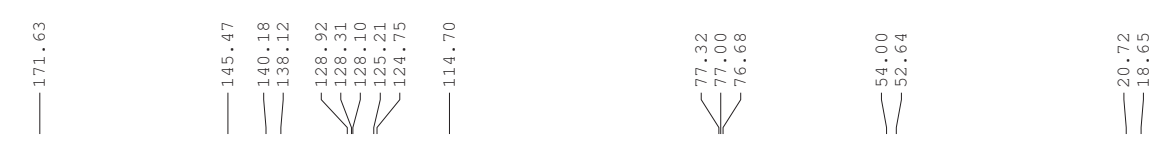<smiles>COC(=O)C1CCC(c2ccccc2)=NN1c1ccc(Cl)cc1</smiles>

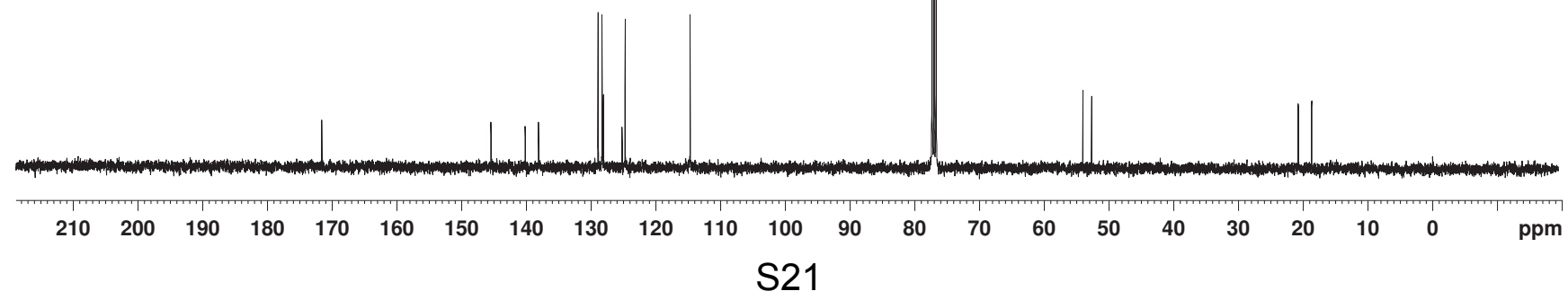




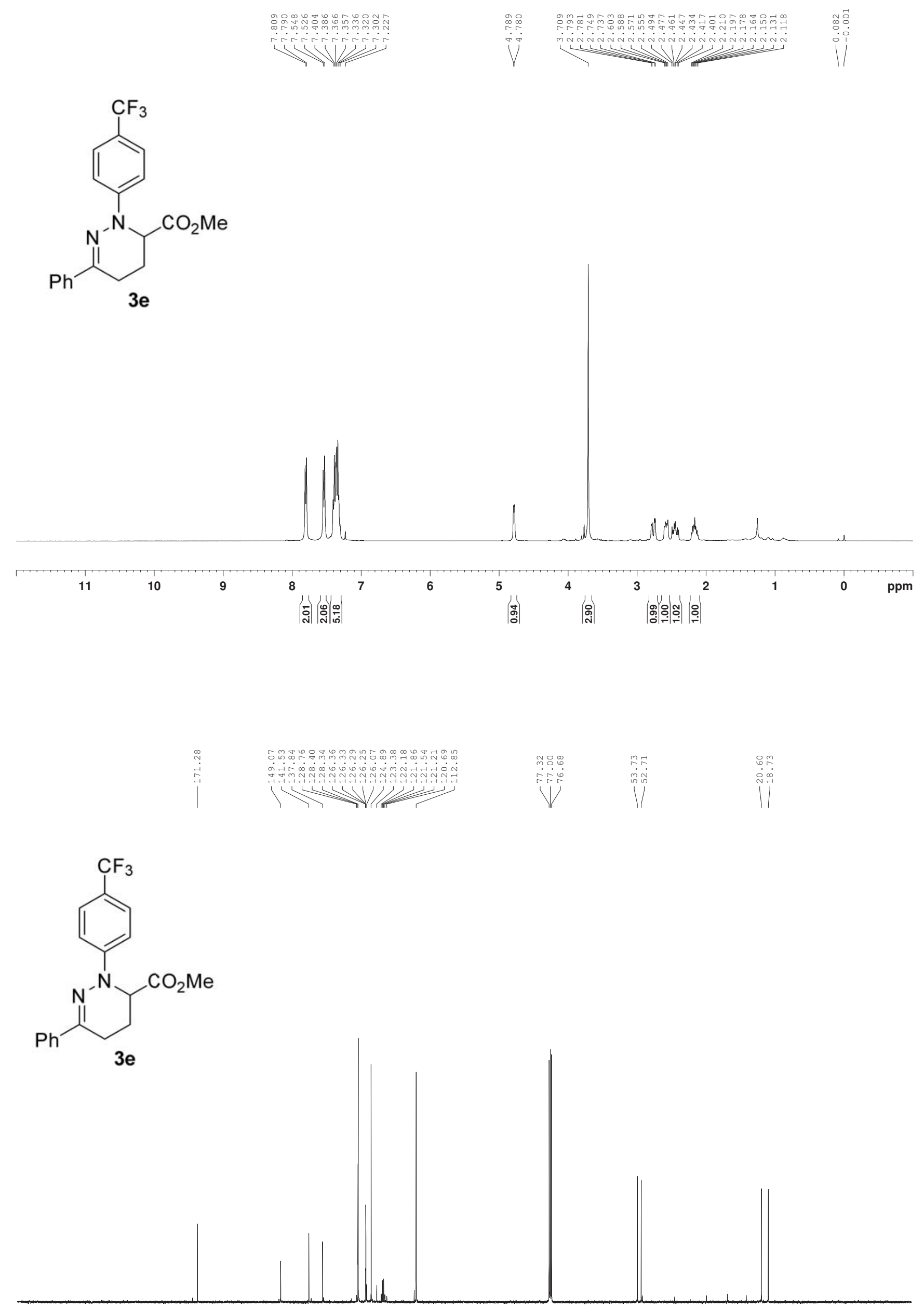

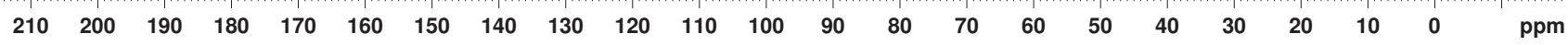


<smiles>COC(=O)C1CCC(c2ccccc2)=NN1c1ccc(C#N)cc1</smiles>

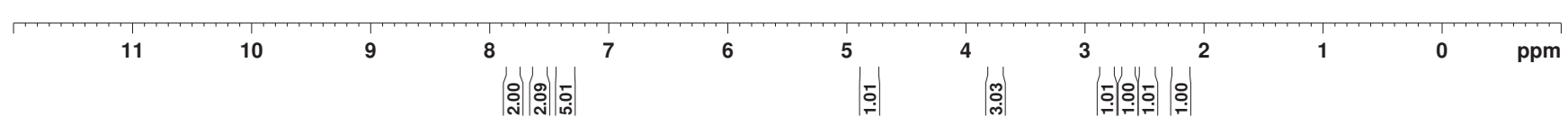
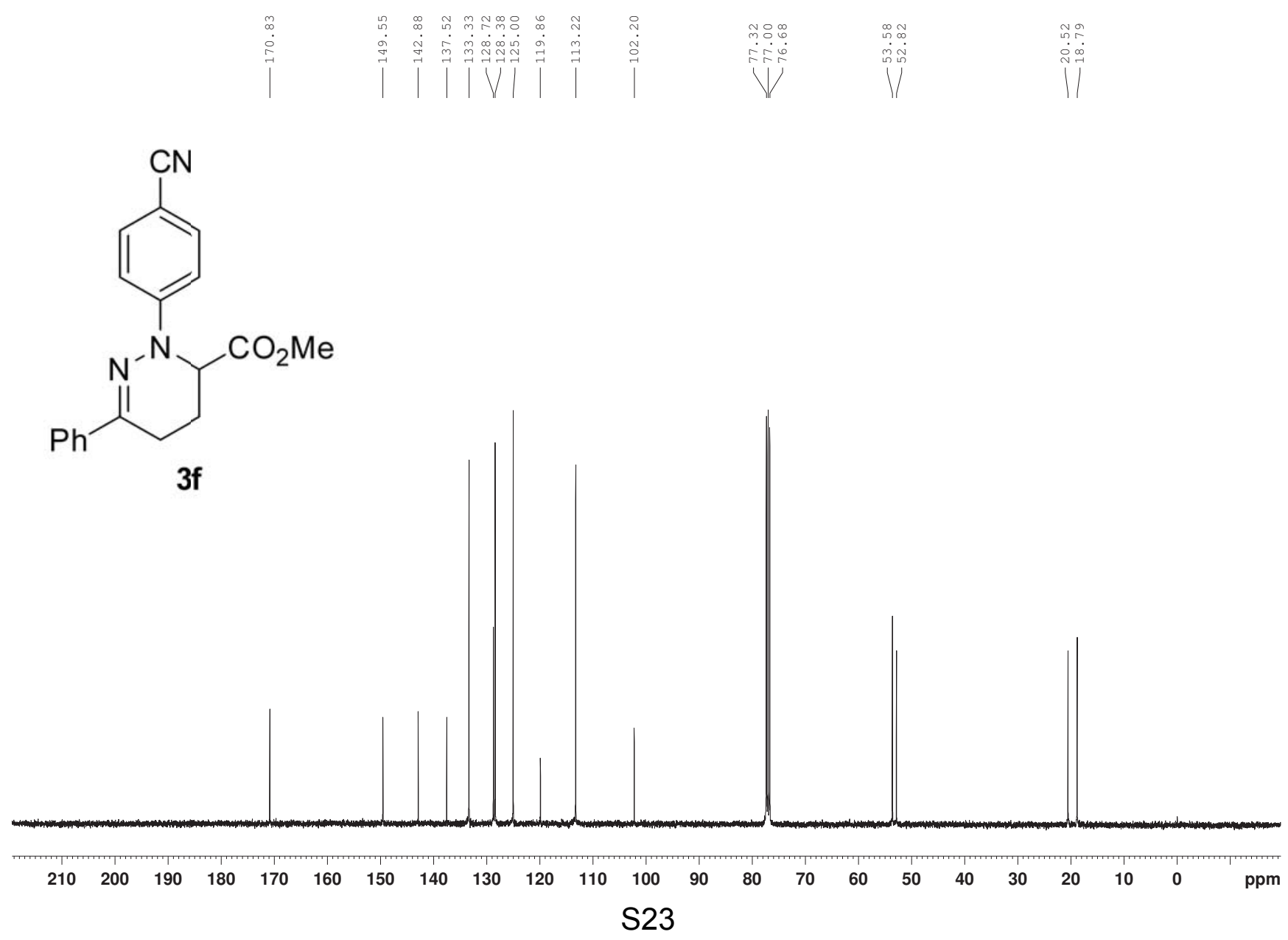
<smiles>COC(=O)C1CCC(c2ccccc2)=NN1c1ccccc1C</smiles>
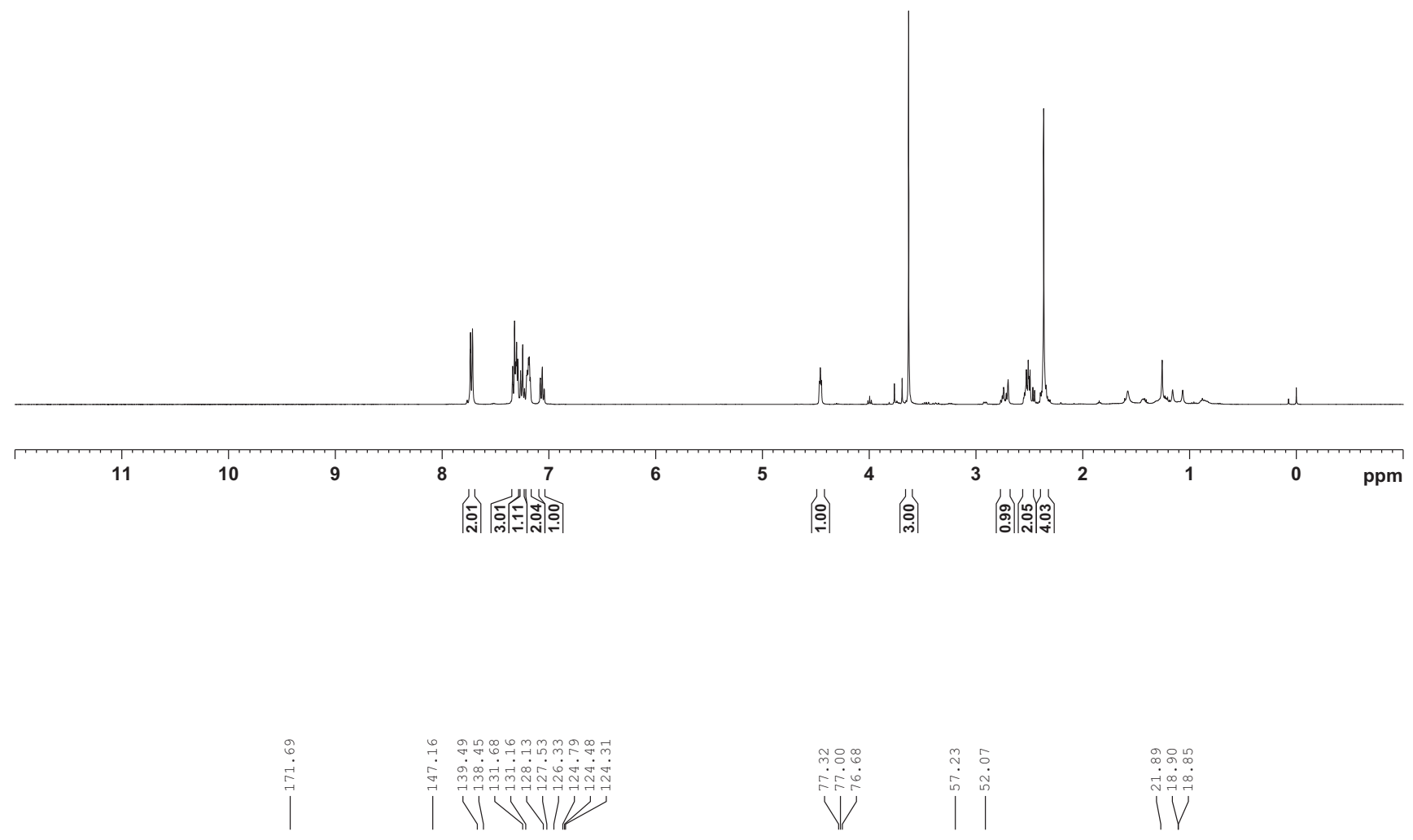<smiles>CC(=O)C1CCC(c2ccccc2)=NN1c1ccccc1C</smiles>

3g

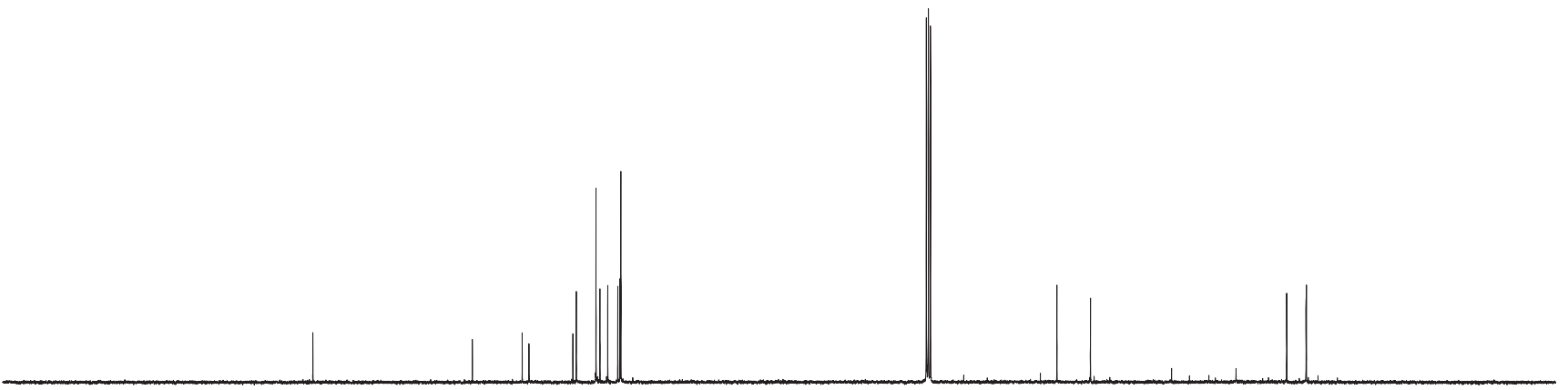




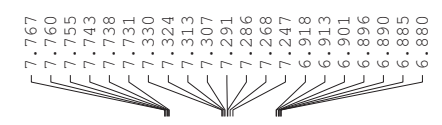<smiles>COC(=O)C1CCC(c2ccc(OC)cc2)=NN1c1ccccc1</smiles>

3j

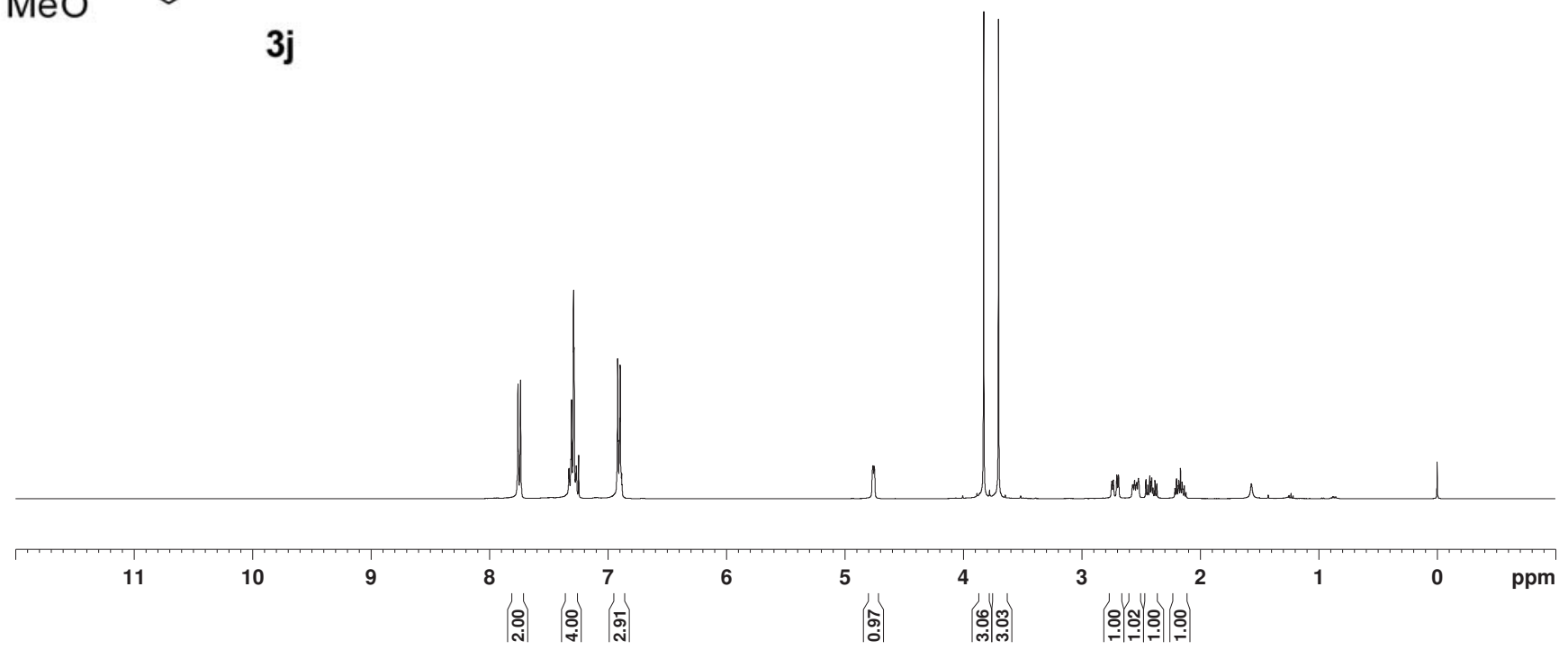<smiles>COc1ccc(C2=NN(c3ccccc3)C(C(C)=O)CC2)cc1</smiles>

3j

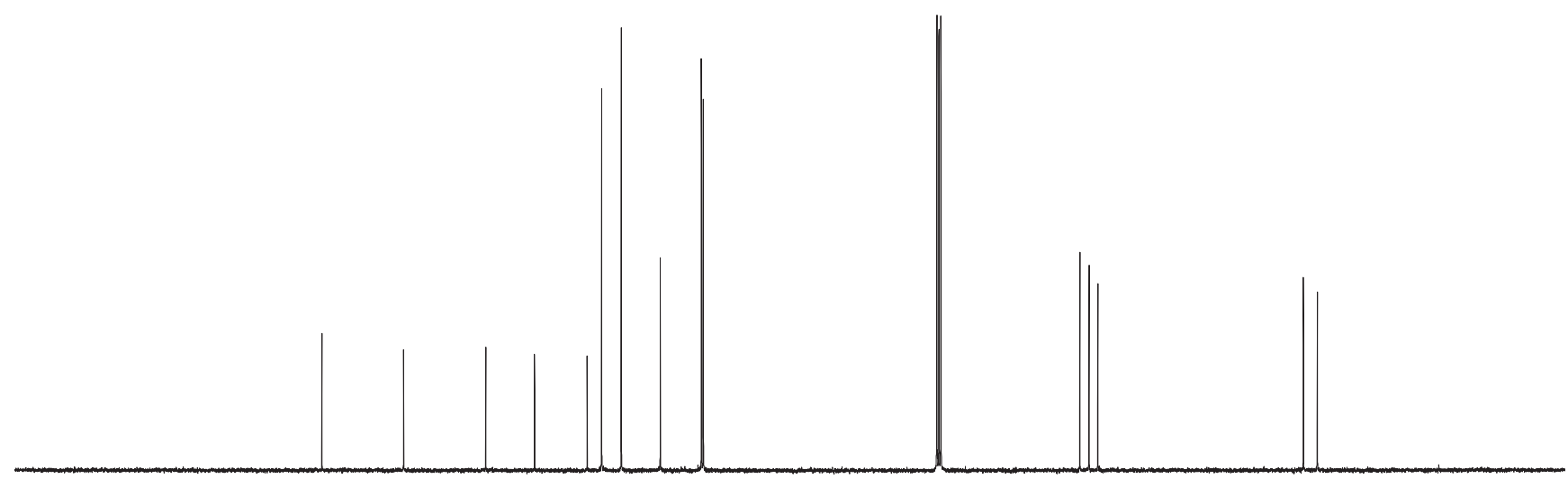


<smiles>CC(=O)C1CCC(c2ccc([N+](=O)[O-])cc2)=NN1c1ccccc1</smiles>

3k
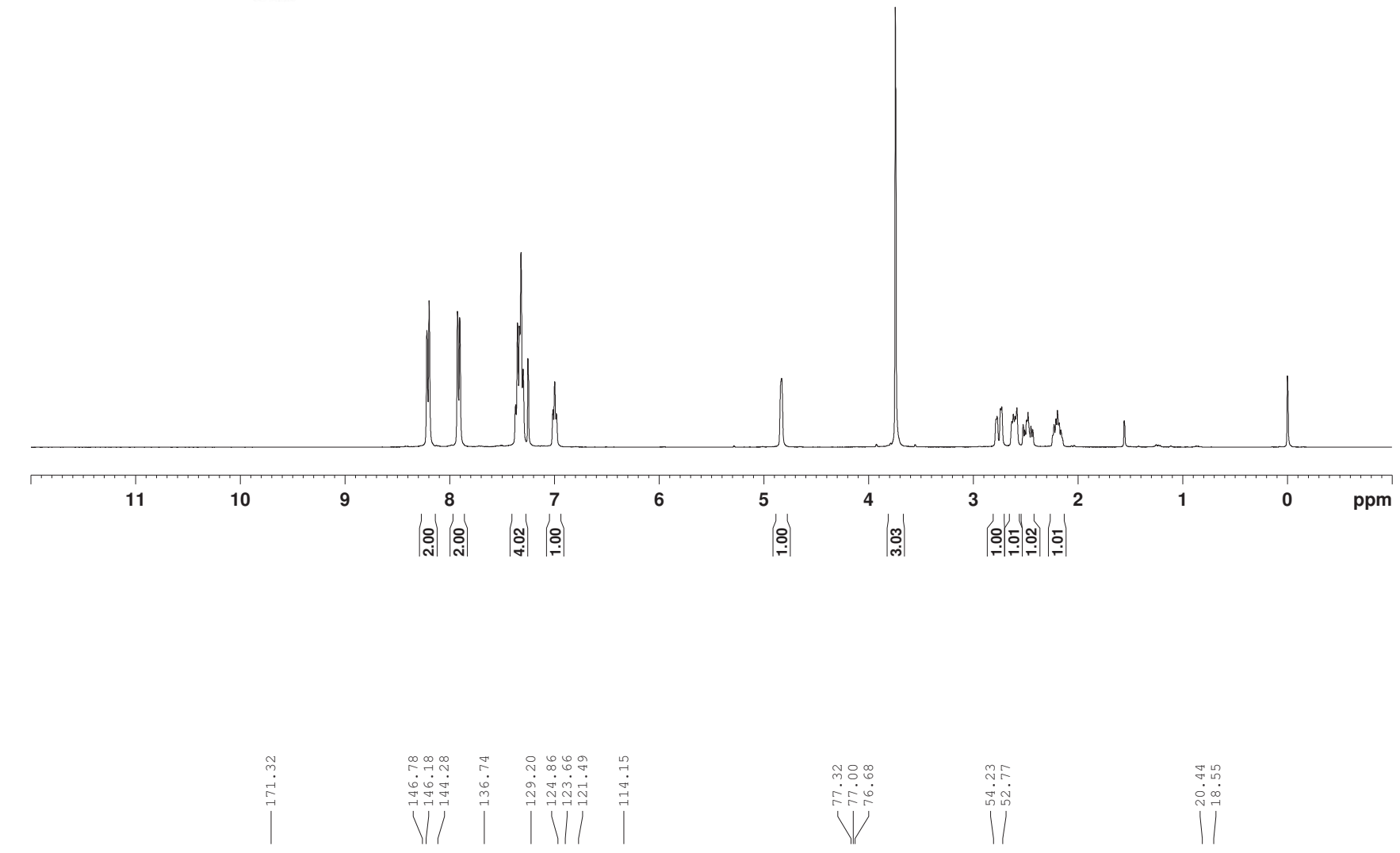<smiles>CC(=O)C1CCC(c2ccc([N+](=O)[O-])cc2)=NN1c1ccccc1</smiles>

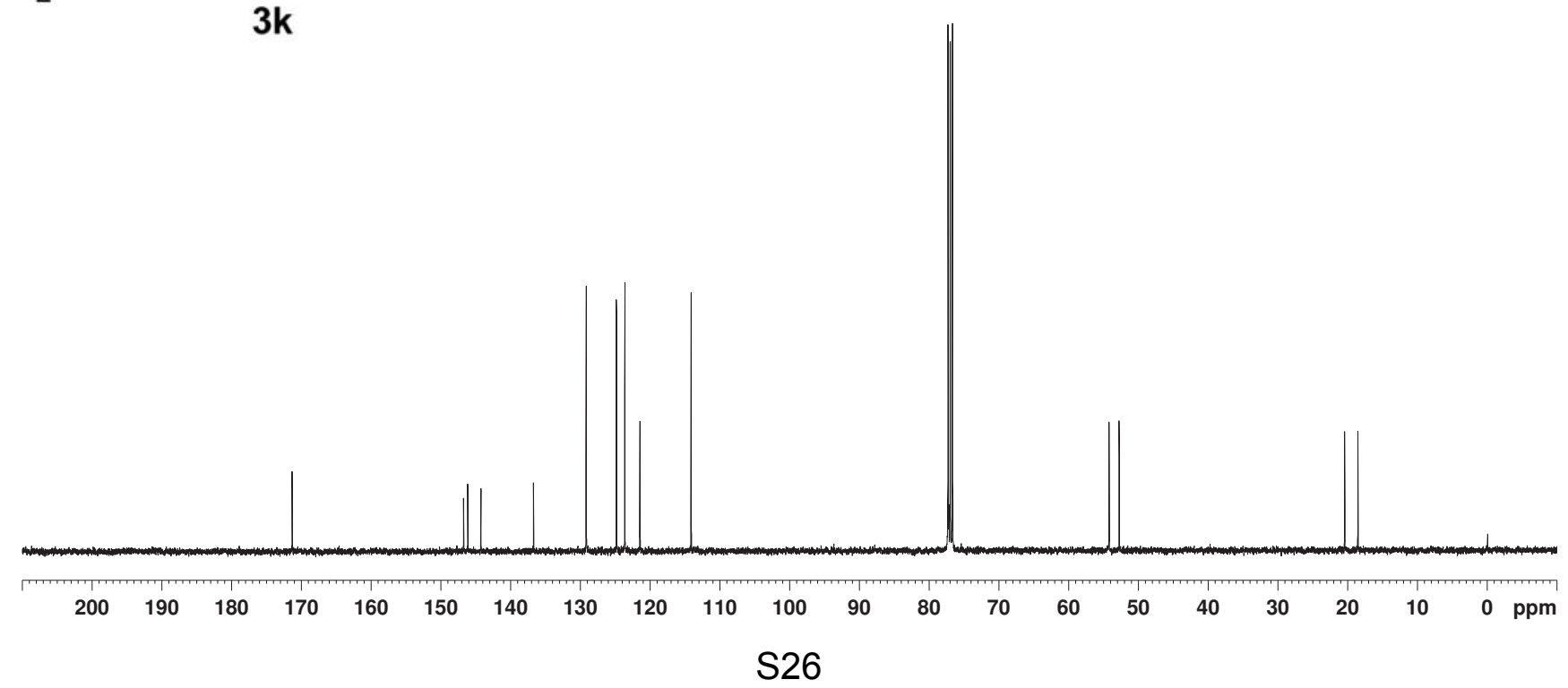


<smiles>CC(=O)C1CCC(c2ccccc2Br)=NN1c1ccccc1</smiles>

3I

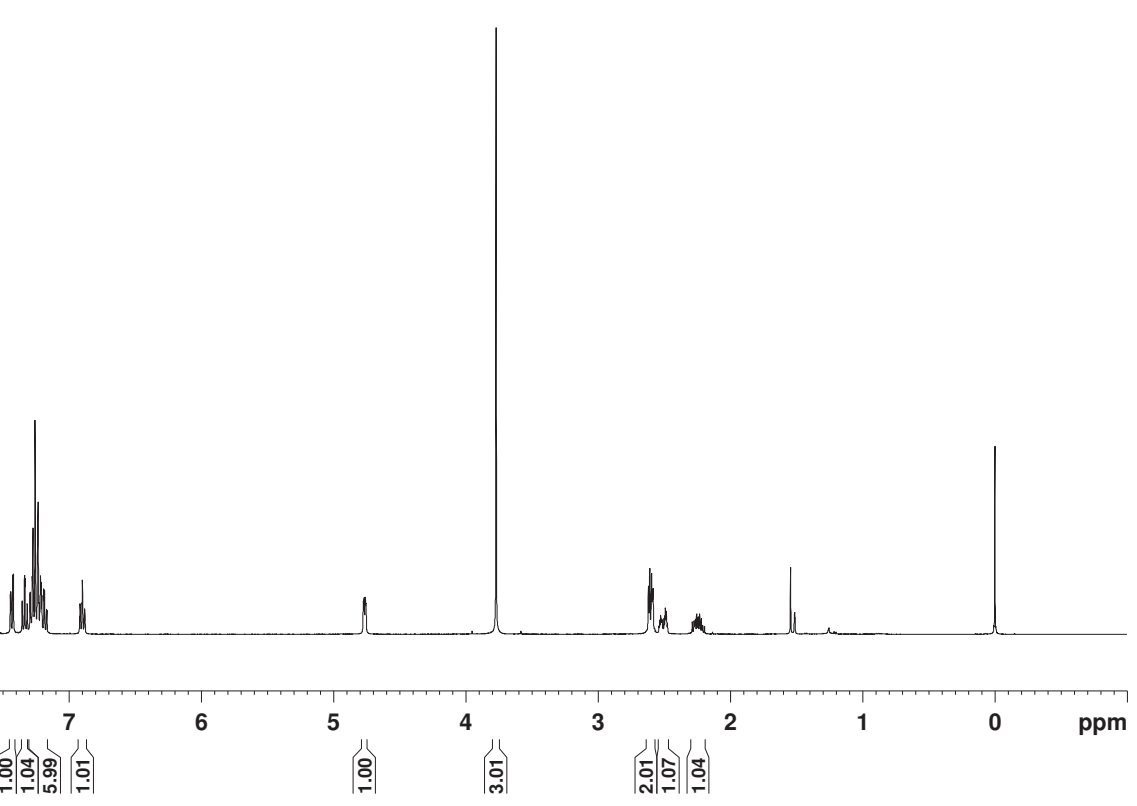<smiles>CC(=O)C1CCC(c2ccccc2Br)=NN1c1ccccc1</smiles>

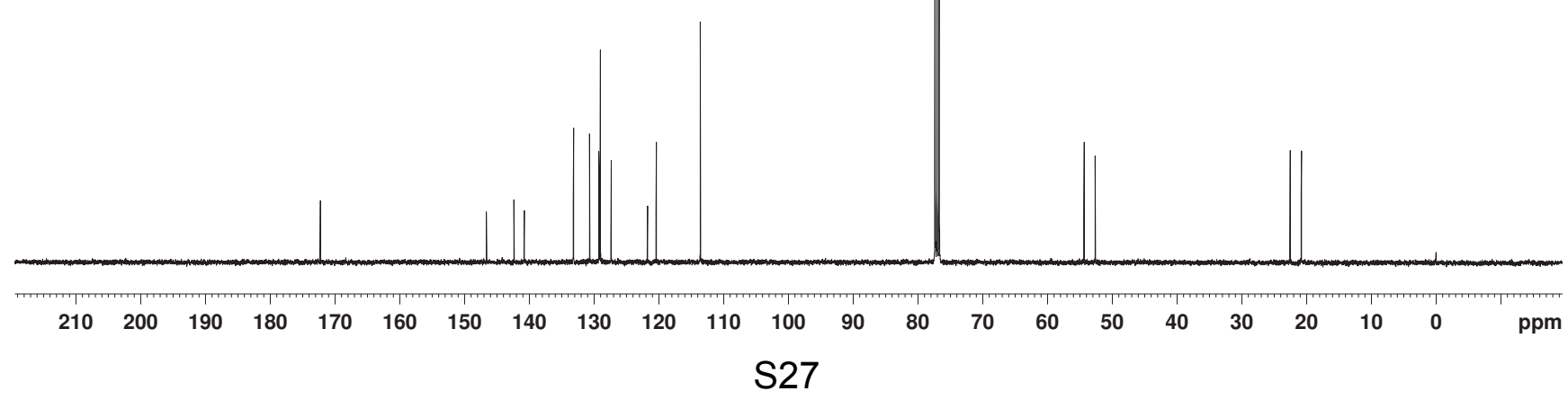



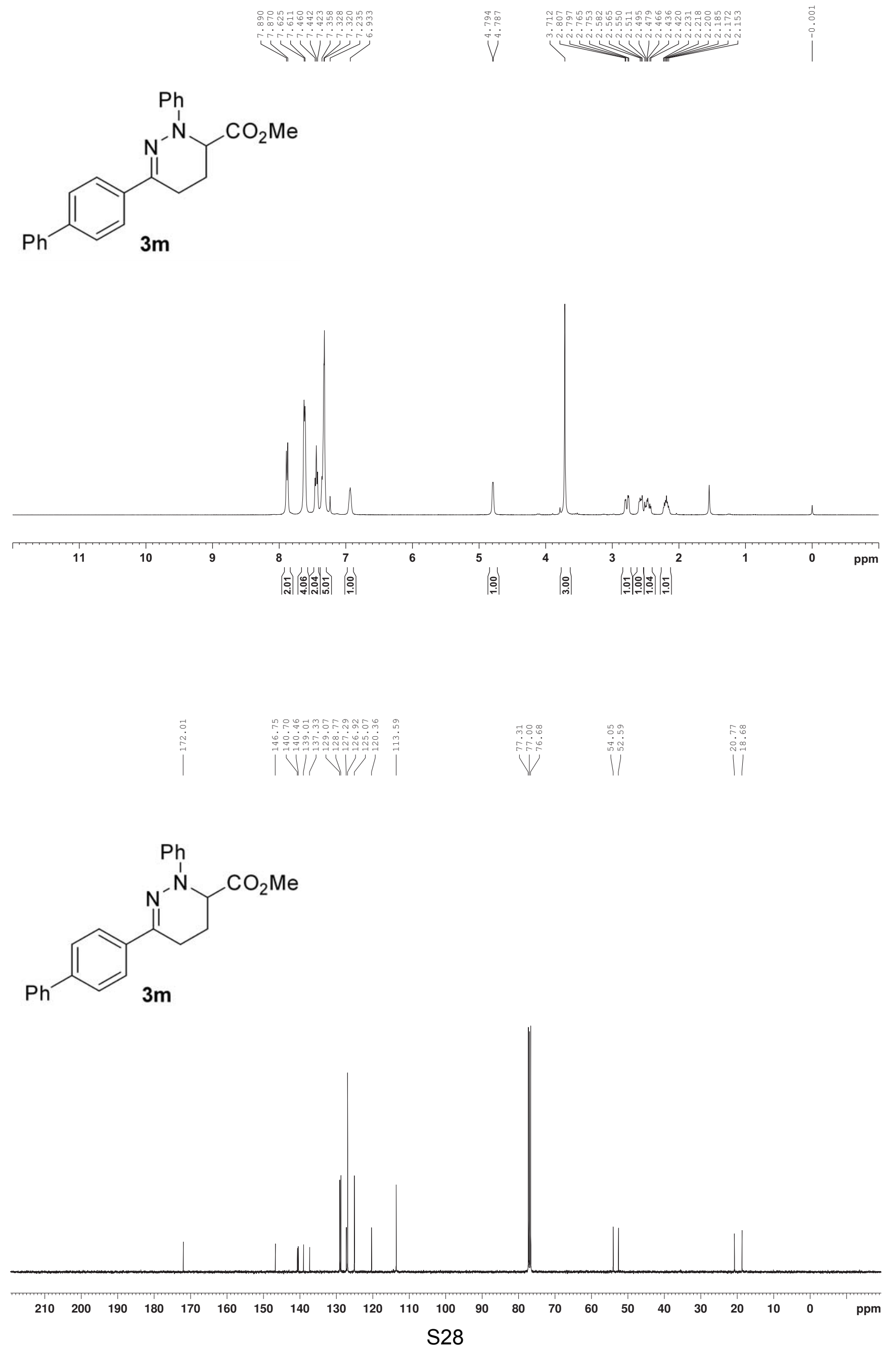

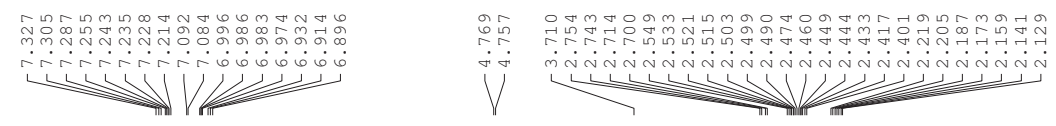<smiles>CC(=O)C1CCC(c2cccs2)=NN1c1ccccc1</smiles>

Nhe

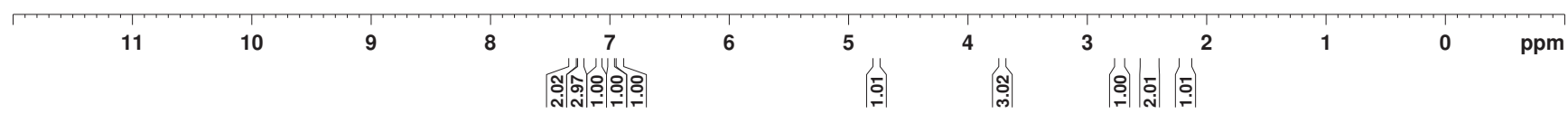

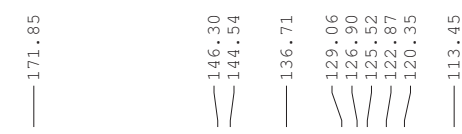
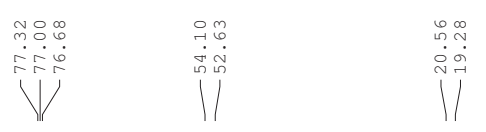<smiles>CC(=O)C1CCC(c2cccs2)=NN1c1ccccc1</smiles> 
<smiles>COC(=O)C1CCC(C)=NN1c1ccccc1</smiles>

30
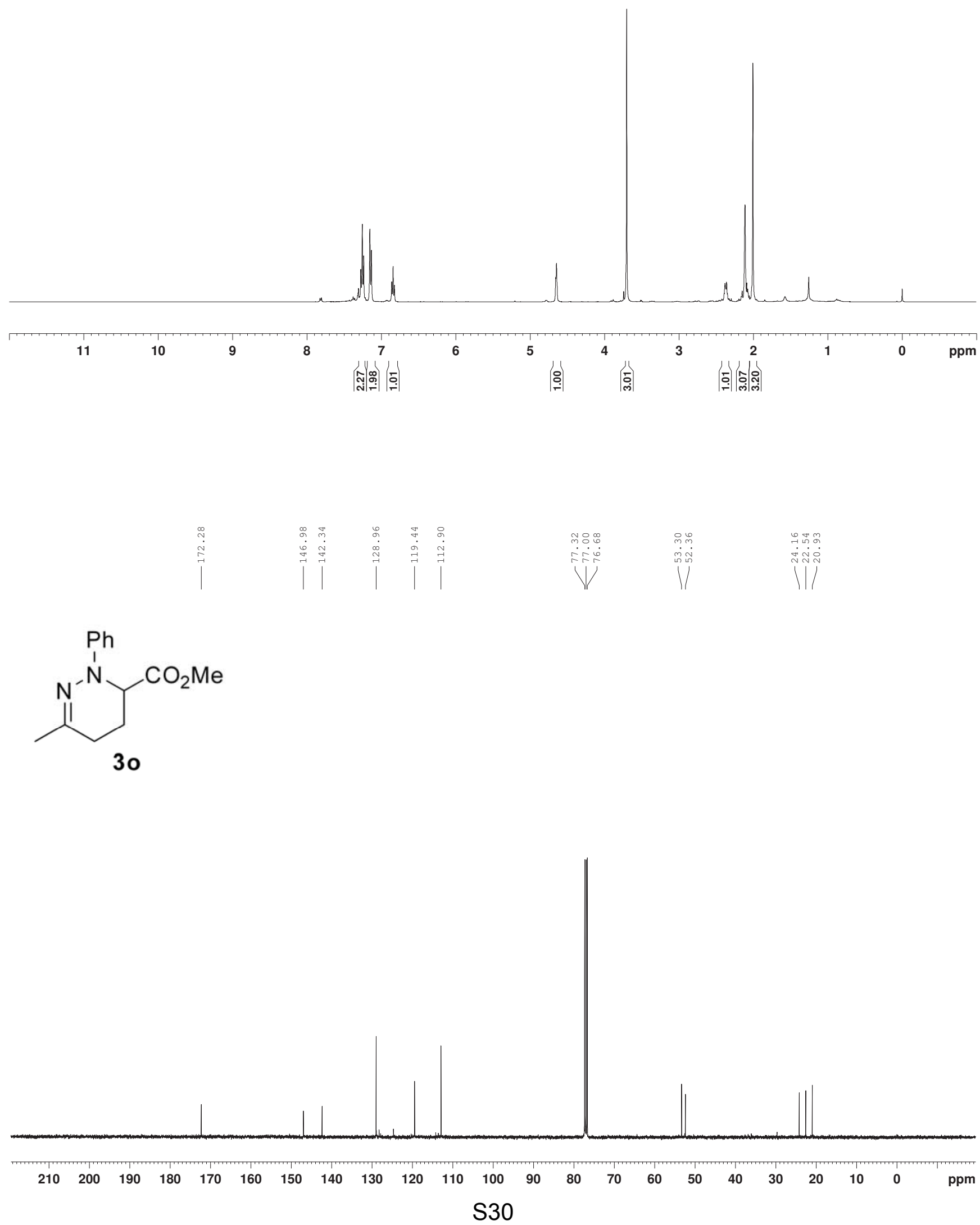

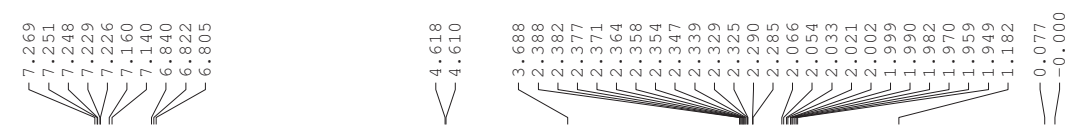<smiles>CC(=O)C1CCC(C(C)(C)C)=NN1c1ccccc1</smiles>
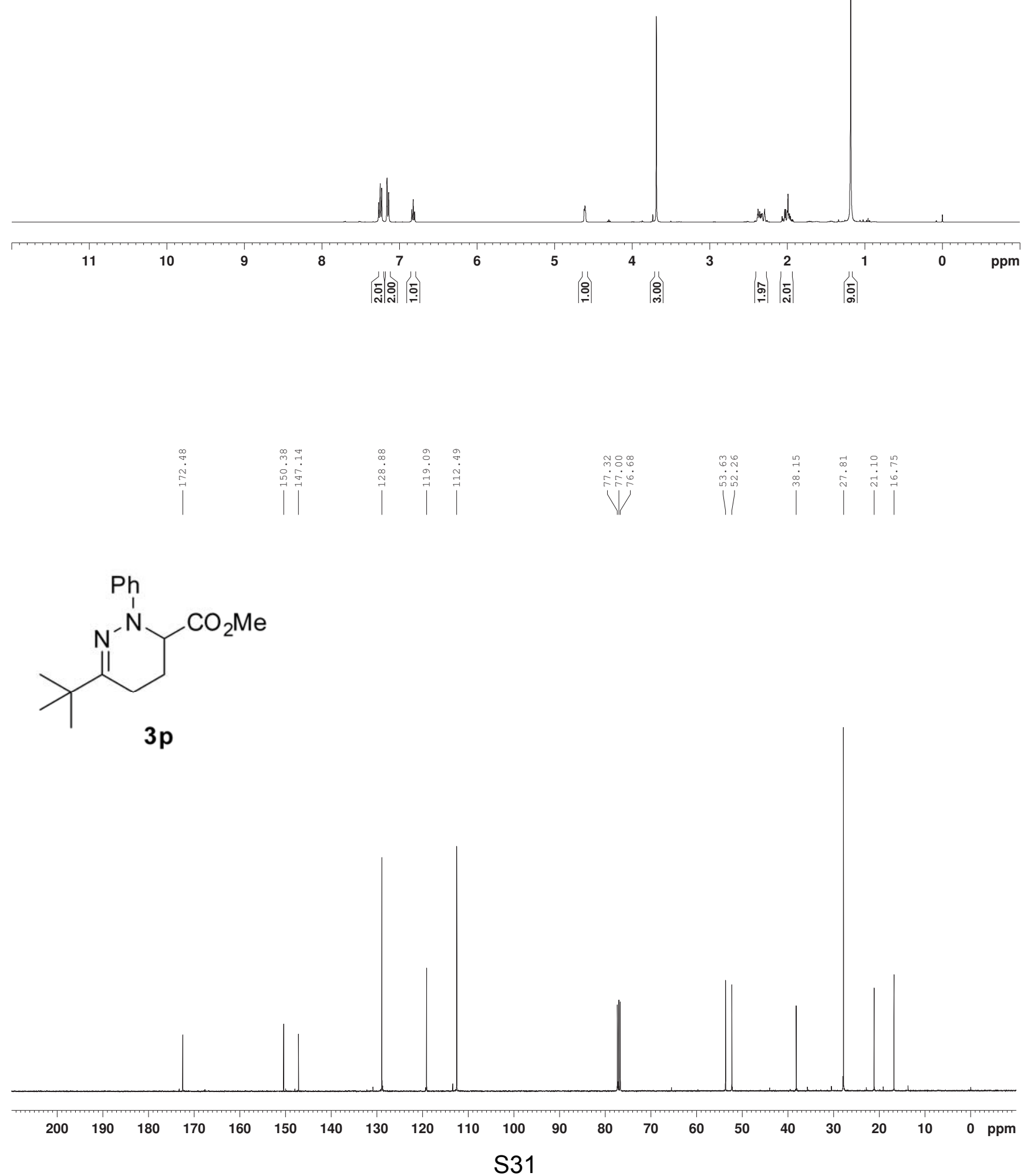
<smiles>CCOC(=O)C1CCC(c2ccccc2)=NN1c1ccccc1</smiles>

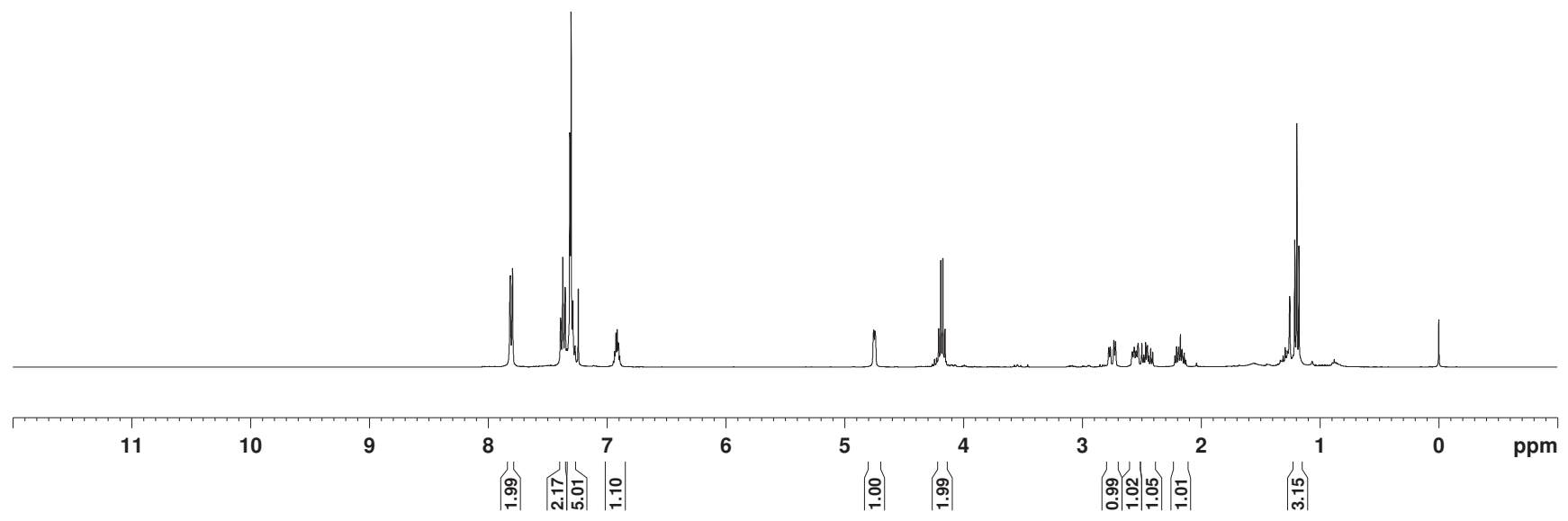<smiles>CCOC(=O)C1CCC(c2ccccc2)=NN1c1ccccc1</smiles>
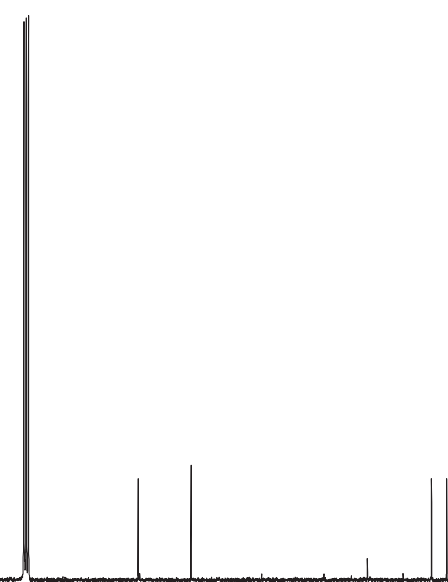
<smiles>CCCOC(=O)C1CCC(c2ccccc2)=NN1c1ccccc1</smiles>

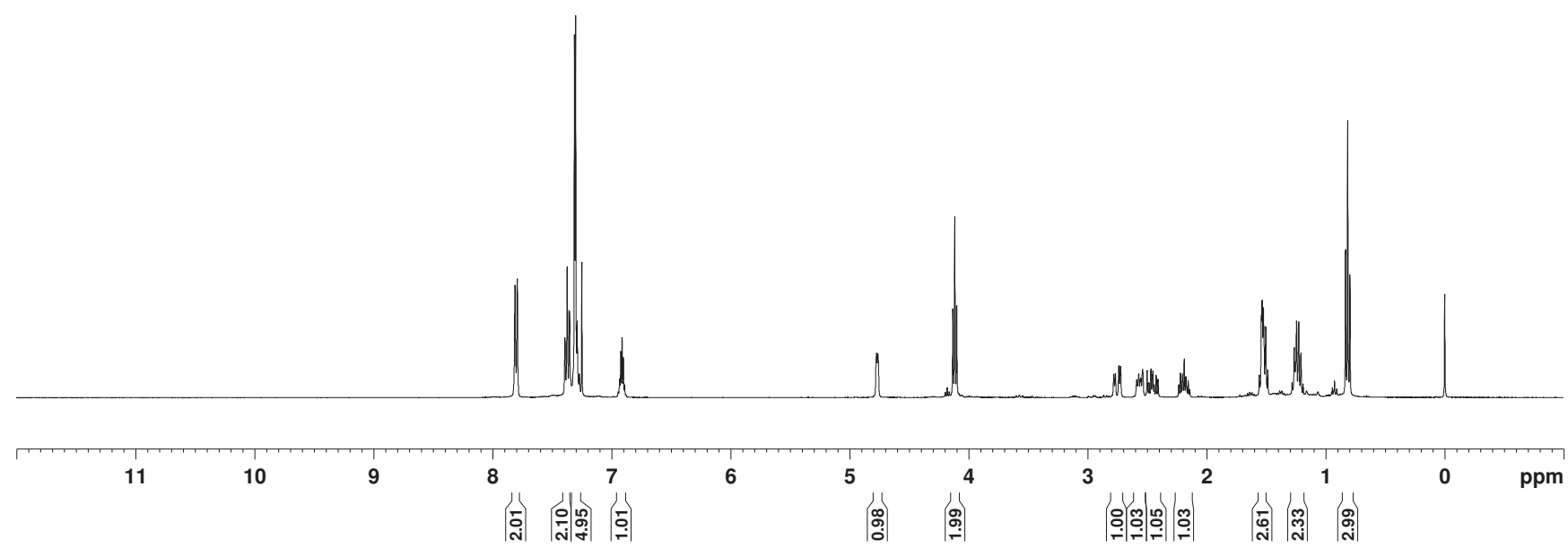

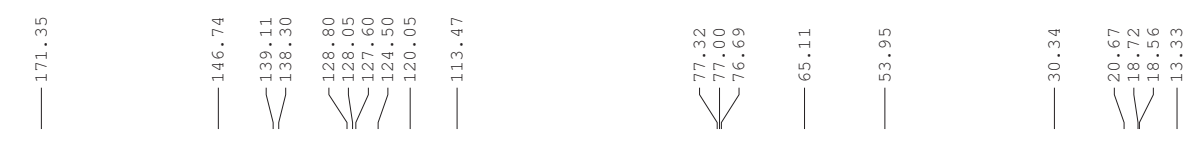<smiles>CCCOC(=O)C1CCC(c2ccccc2)=NN1c1ccccc1</smiles>

3s

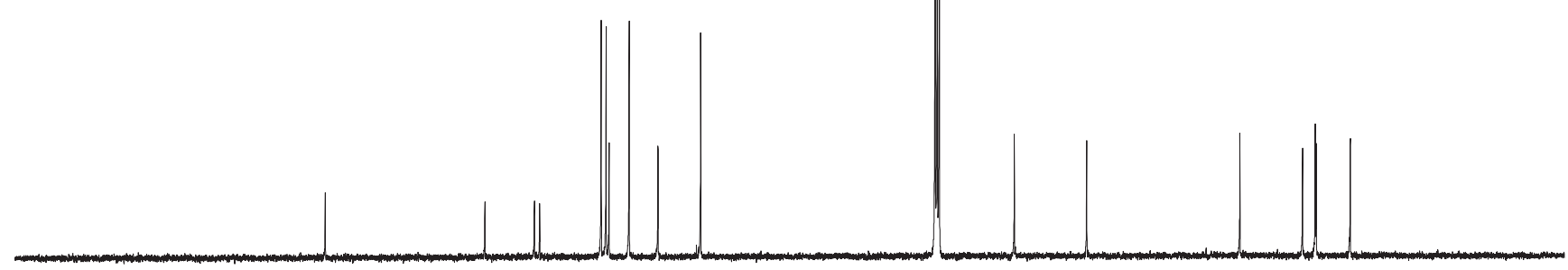

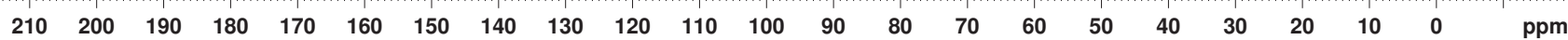




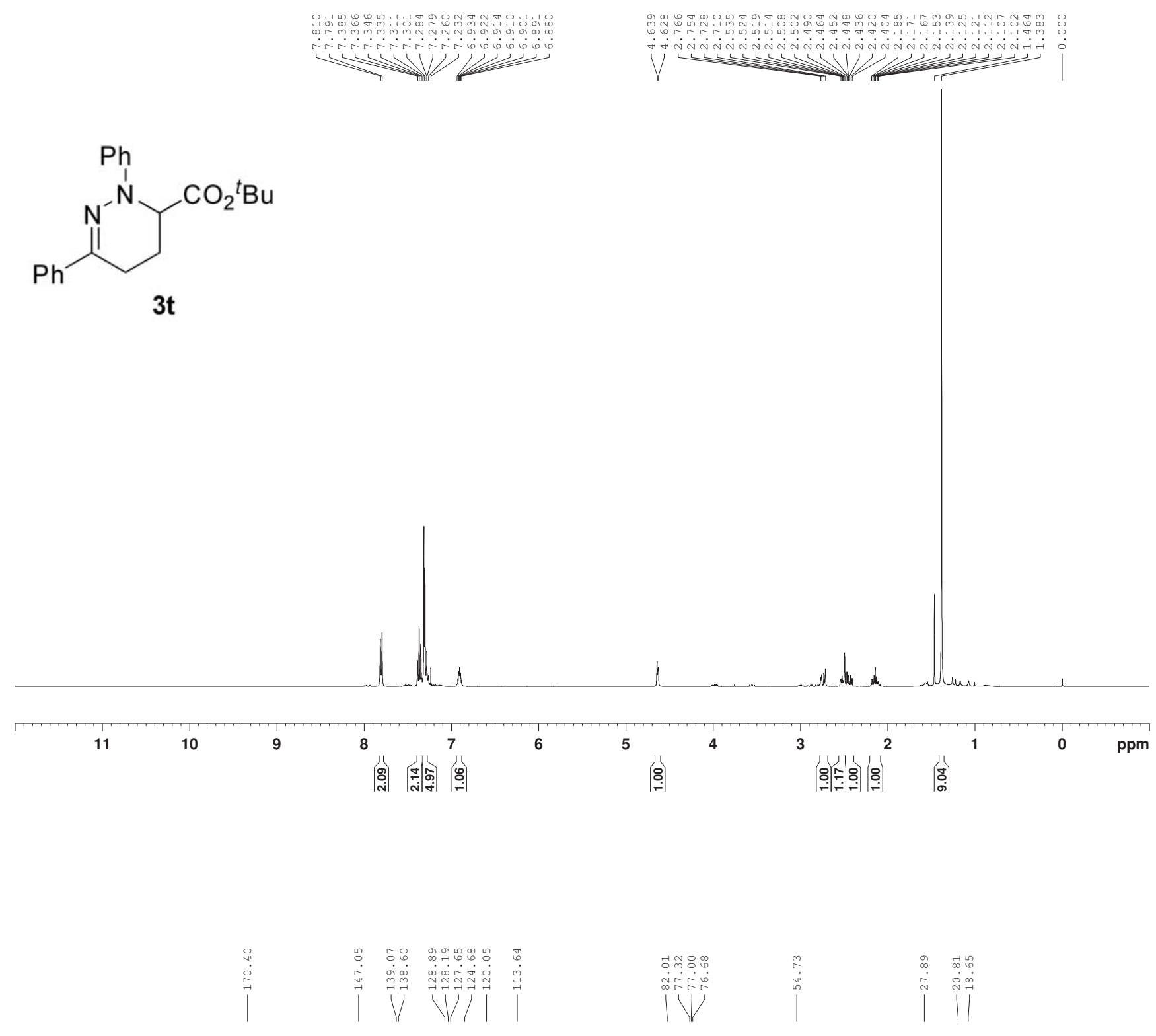<smiles>CCCOC(=O)C1CCC(c2ccccc2)=NN1c1ccccc1</smiles>

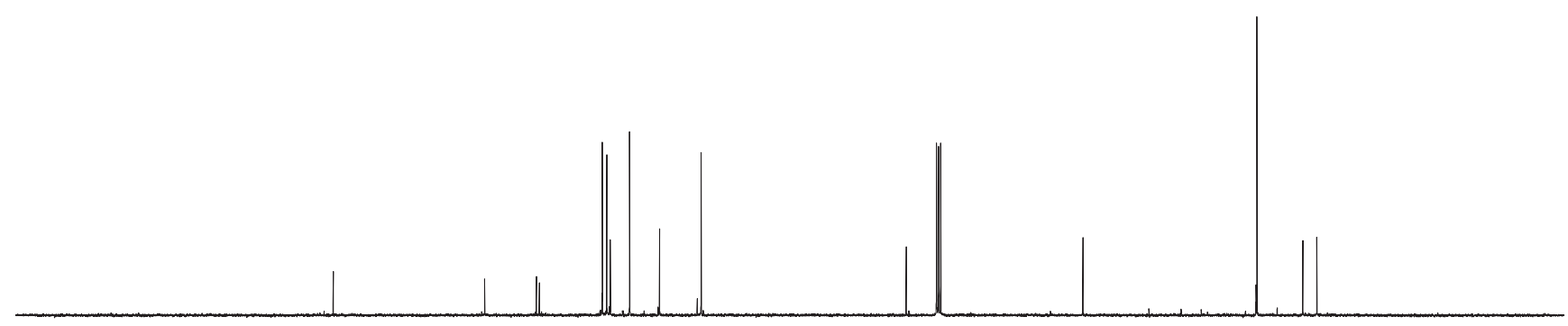


<smiles>O=C(Oc1ccccc1)C1CCC(c2ccccc2)=NN1c1ccccc1</smiles>
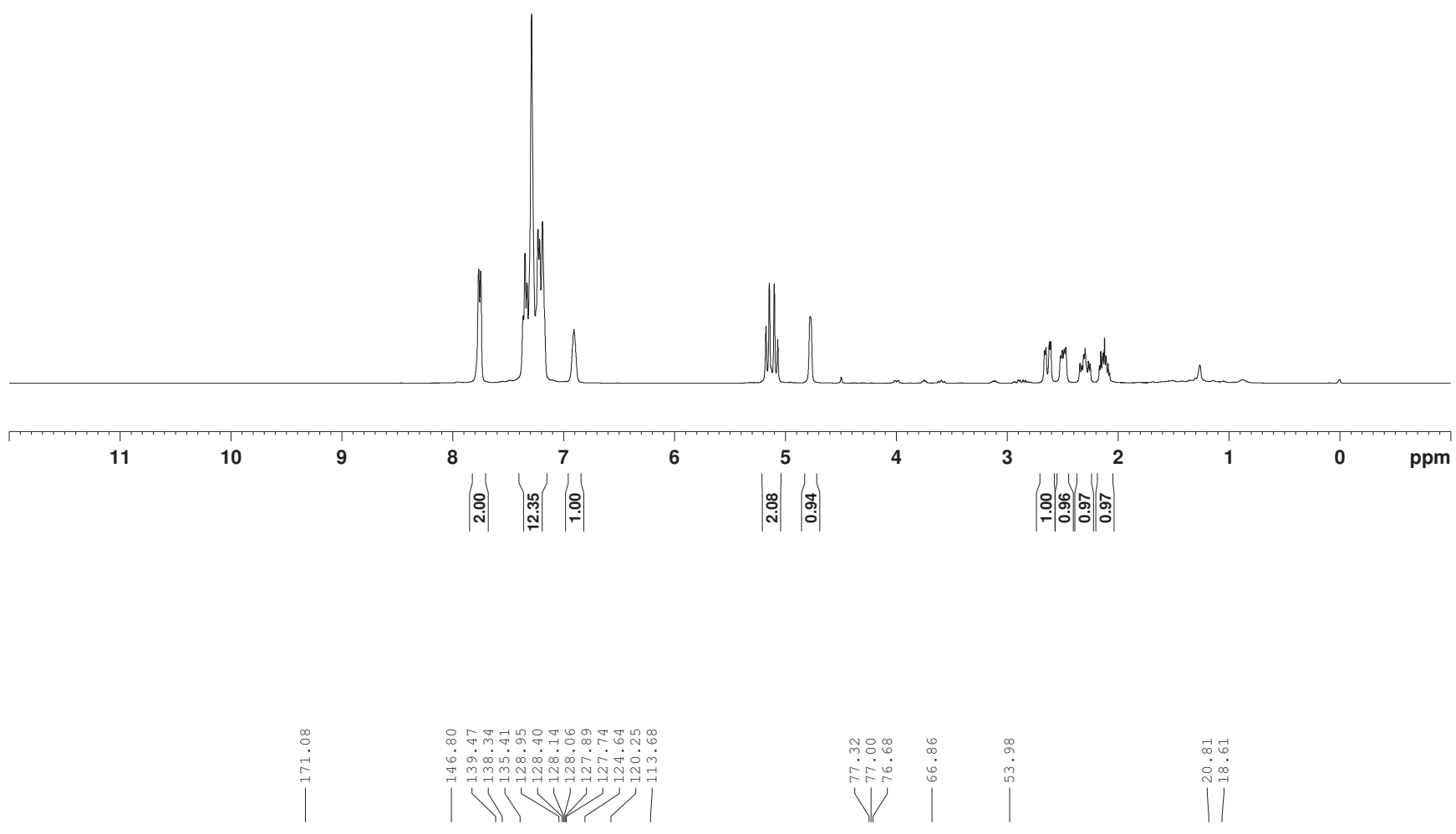<smiles>O=C(Oc1ccccc1)C1CCC(c2ccccc2)=NN1c1ccccc1</smiles>

$3 u$

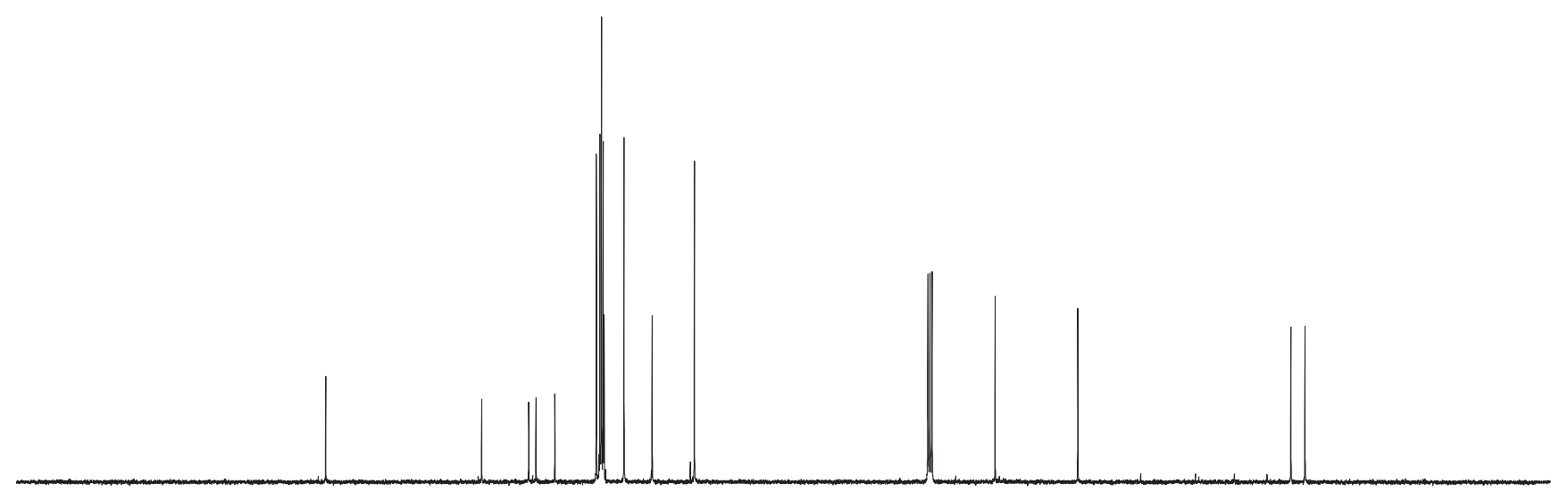




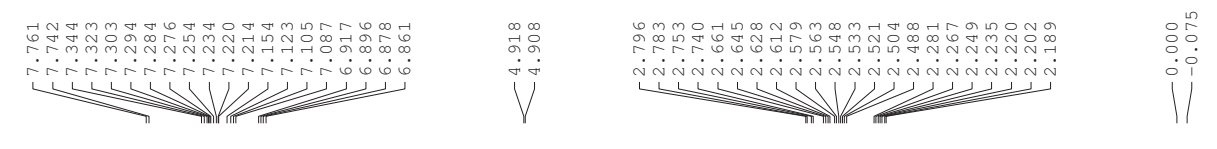<smiles>O=C(c1ccccc1)C1CCC(c2ccccc2)=NN1c1ccccc1</smiles>
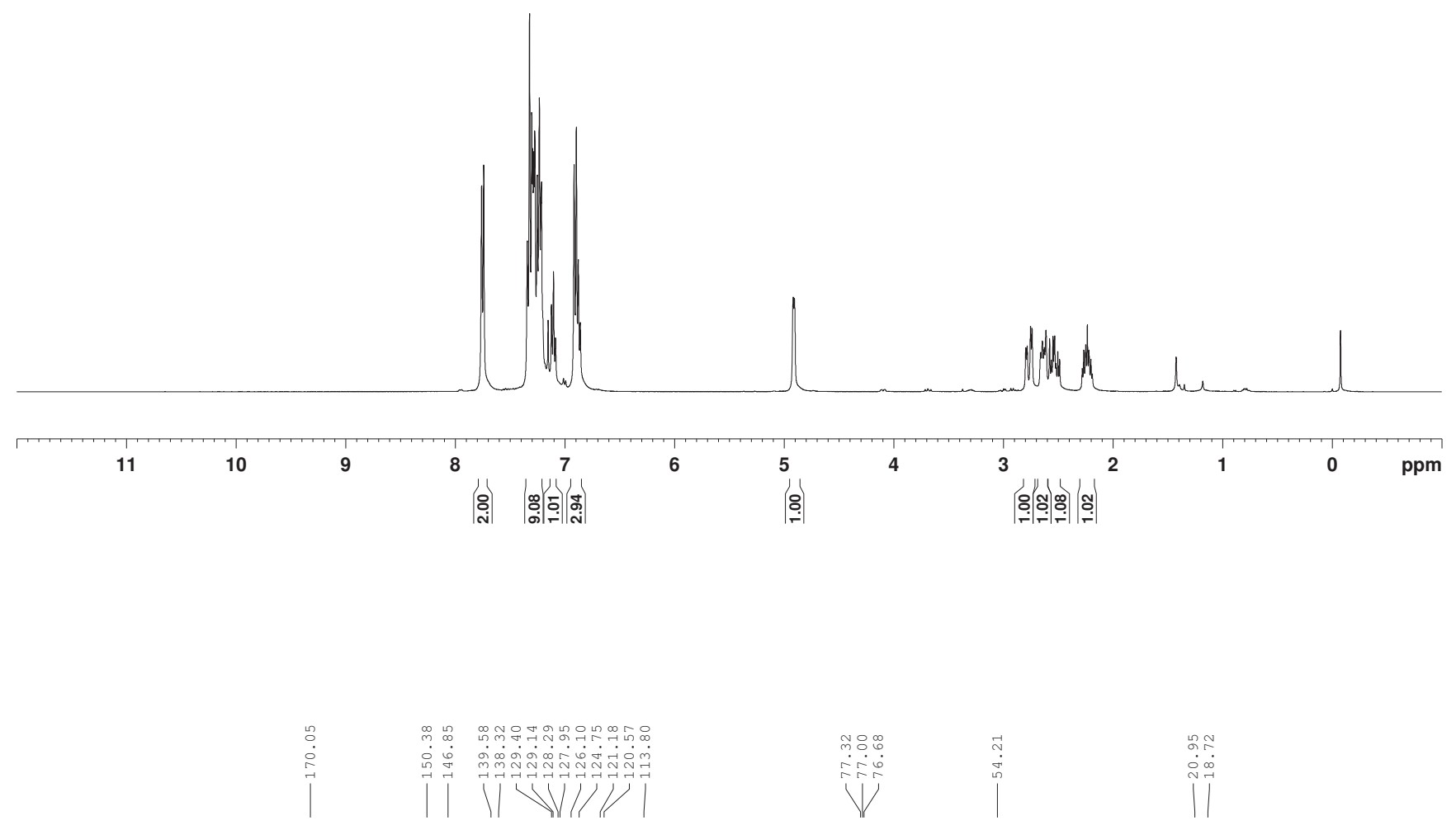<smiles>O=C(c1ccccc1)C(c1ccccc1)C1CCC(c2ccccc2)=NN1c1ccccc1</smiles>

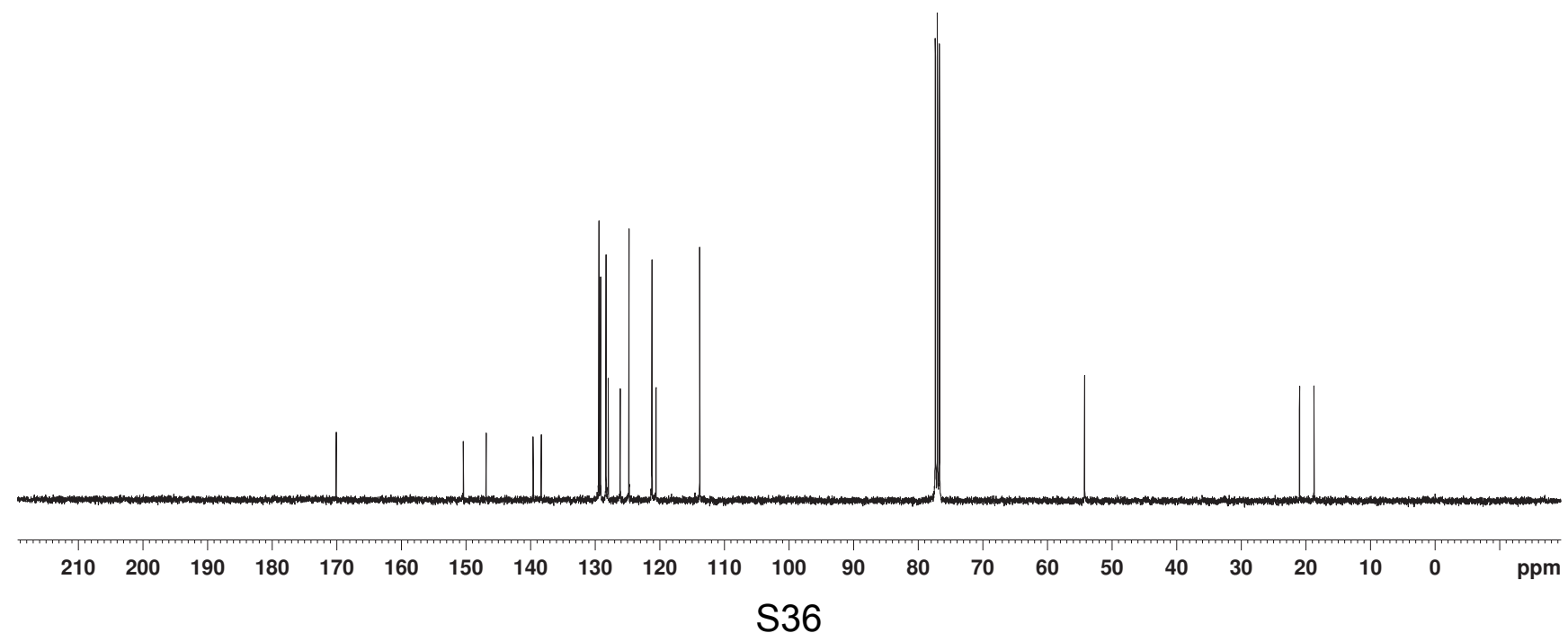




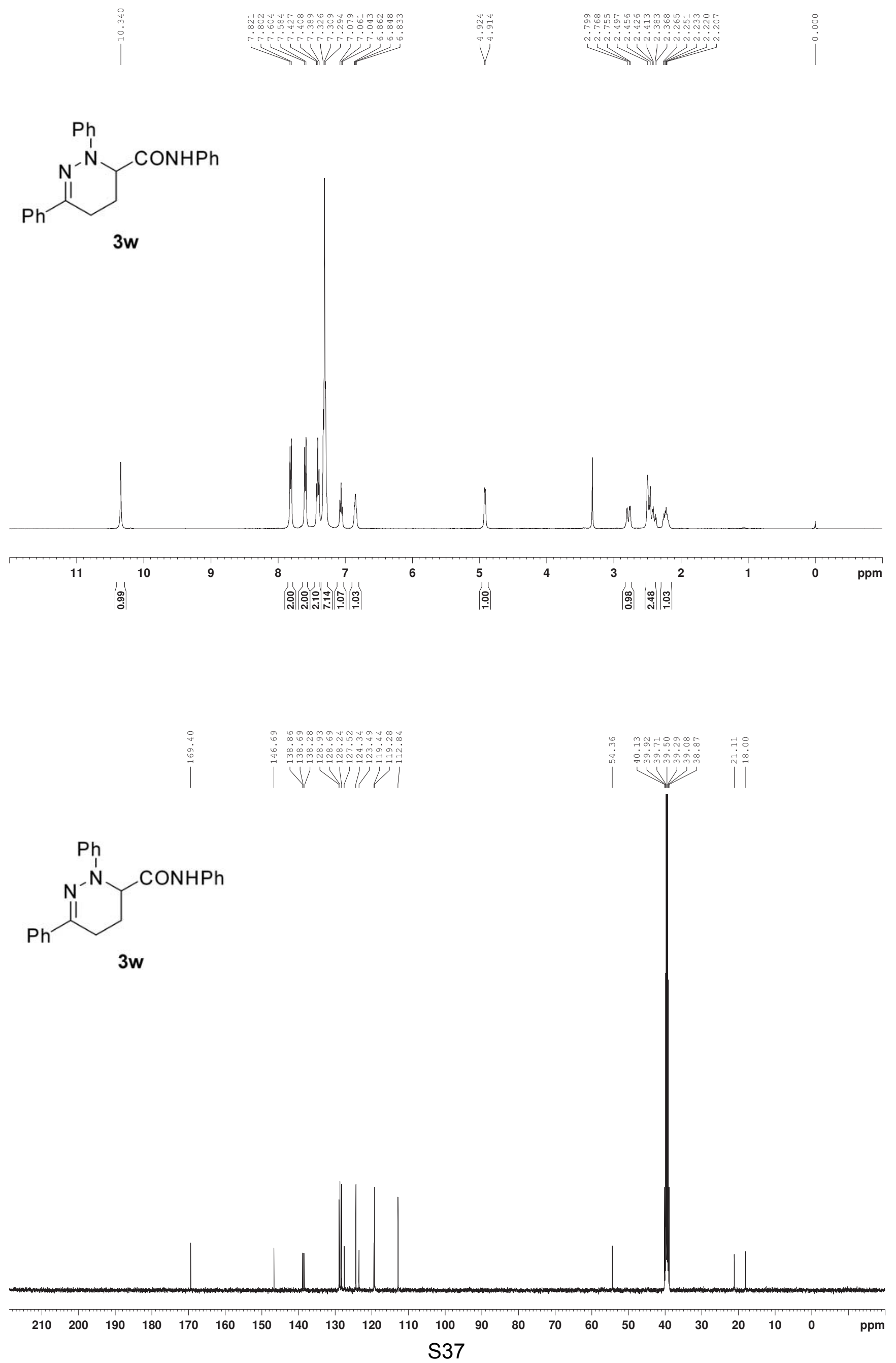



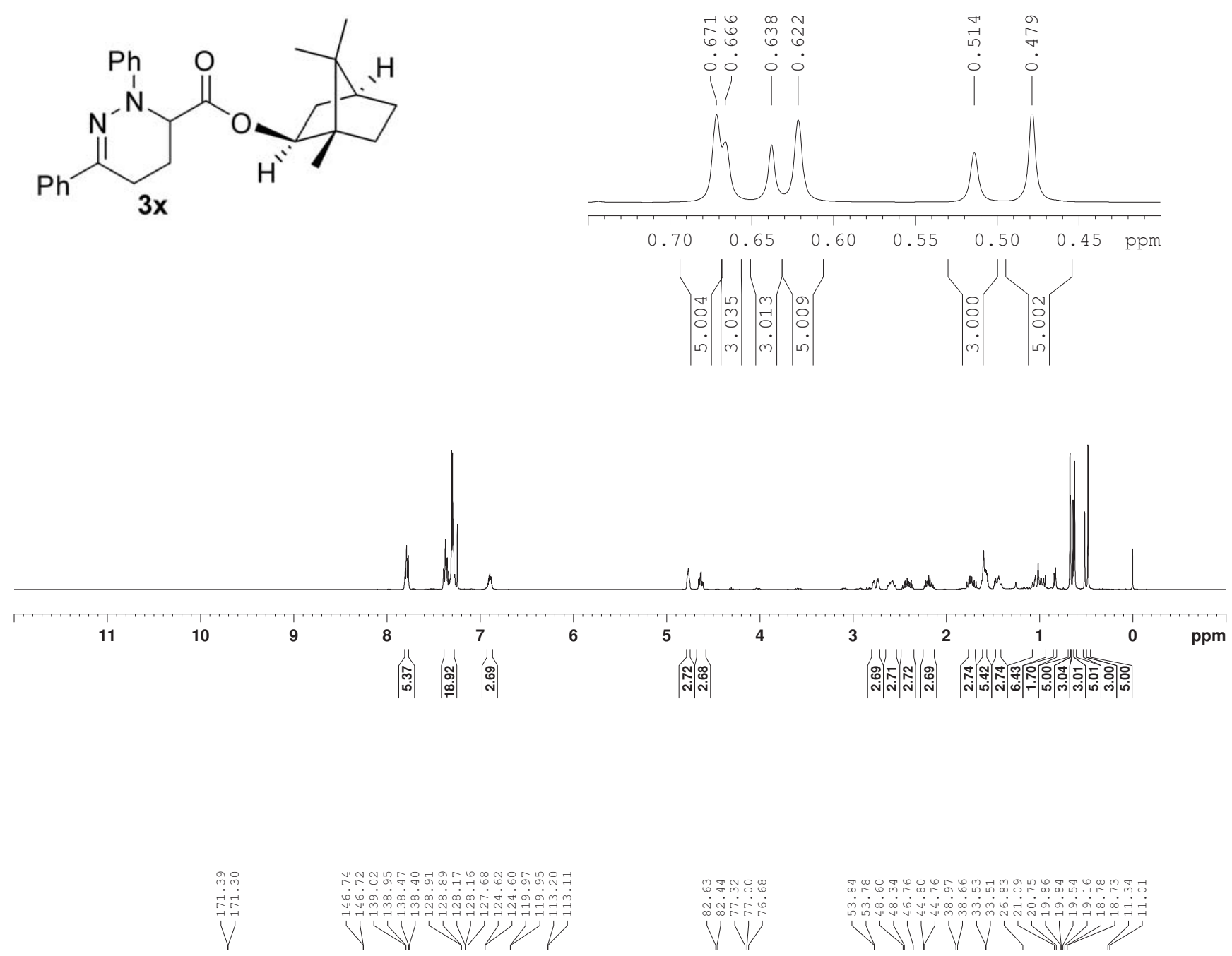<smiles>CC1(C)[C@H]2CC[C@@]1(C)[C@H](OC(=O)C1CCC(c3ccccc3)=NN1c1ccccc1)C2</smiles>

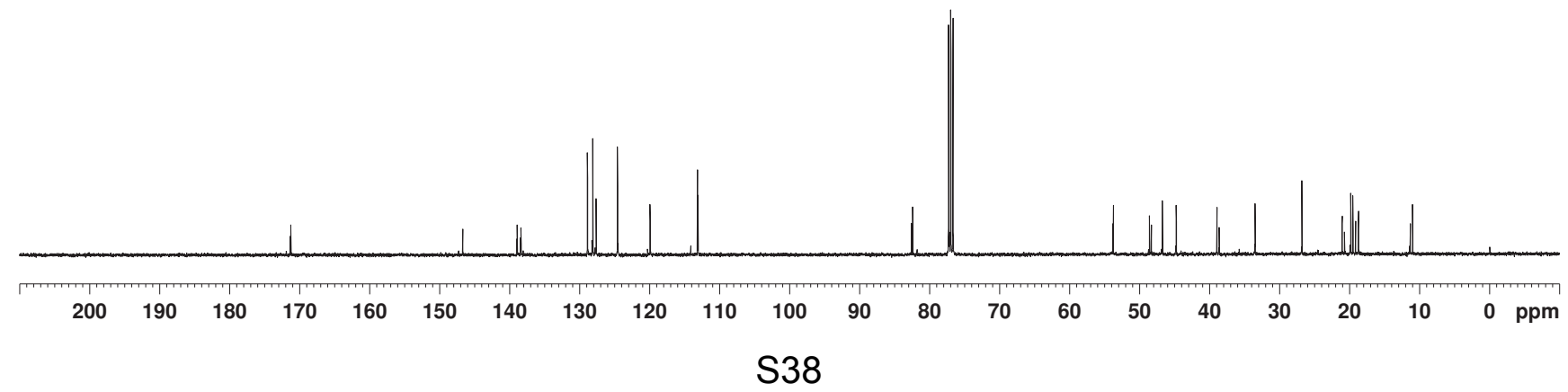



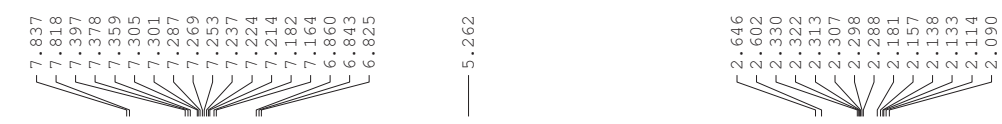<smiles>c1ccc(C2=NN(c3ccccc3)C(c3ccccc3)CC2)cc1</smiles>
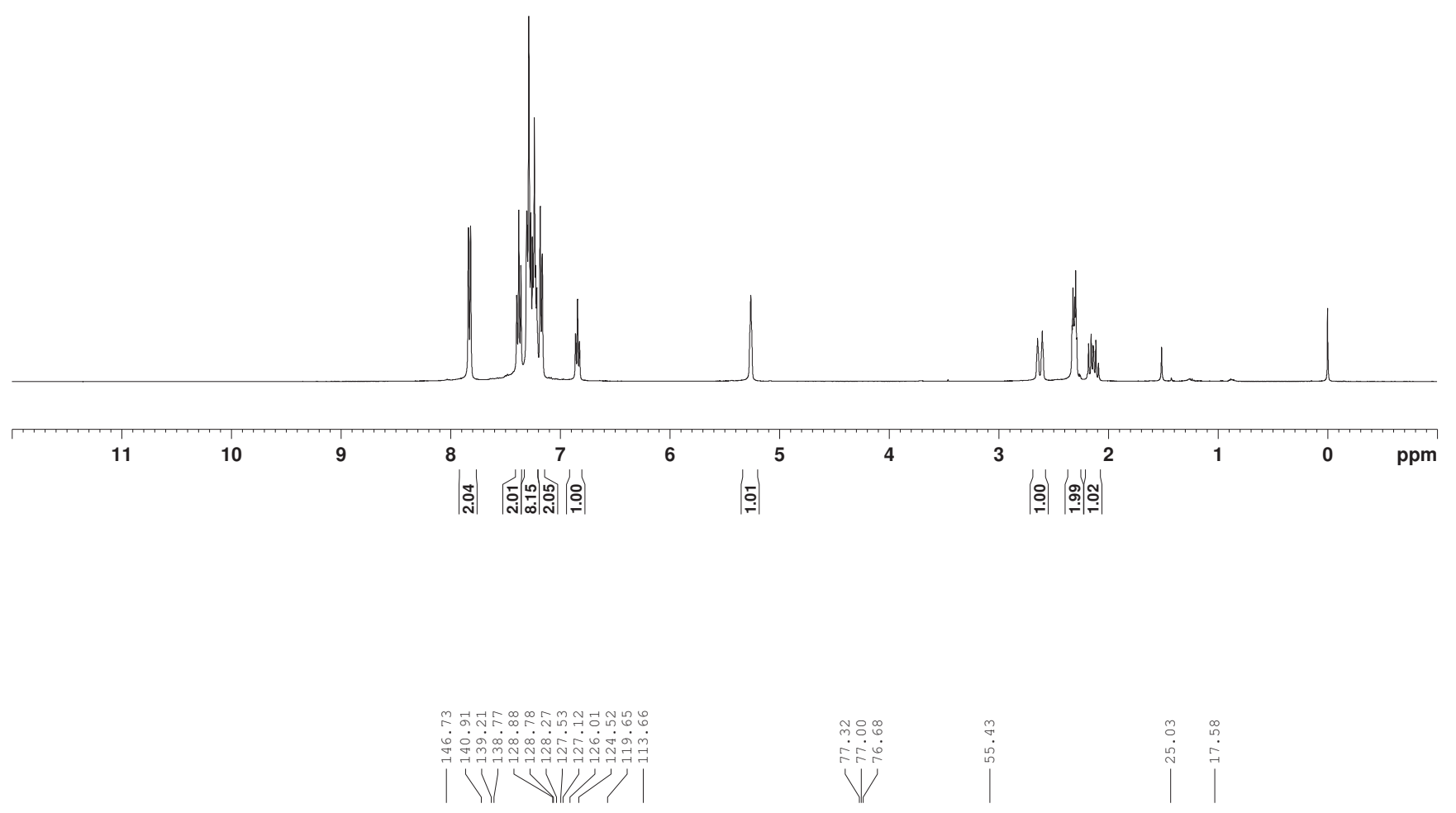<smiles>c1ccc(C2=NN(c3ccccc3)C(c3ccccc3)CC2)cc1</smiles>

$\begin{array}{lllllllllllllllllllllll}210 & 200 & 190 & 180 & 170 & 160 & 150 & 140 & 130 & 120 & 110 & 100 & 90 & 80 & 70 & 60 & 50 & 40 & 30 & 20 & 10 & 0 & \mathrm{ppm}\end{array}$ 
<smiles>Cc1ccccc1</smiles>

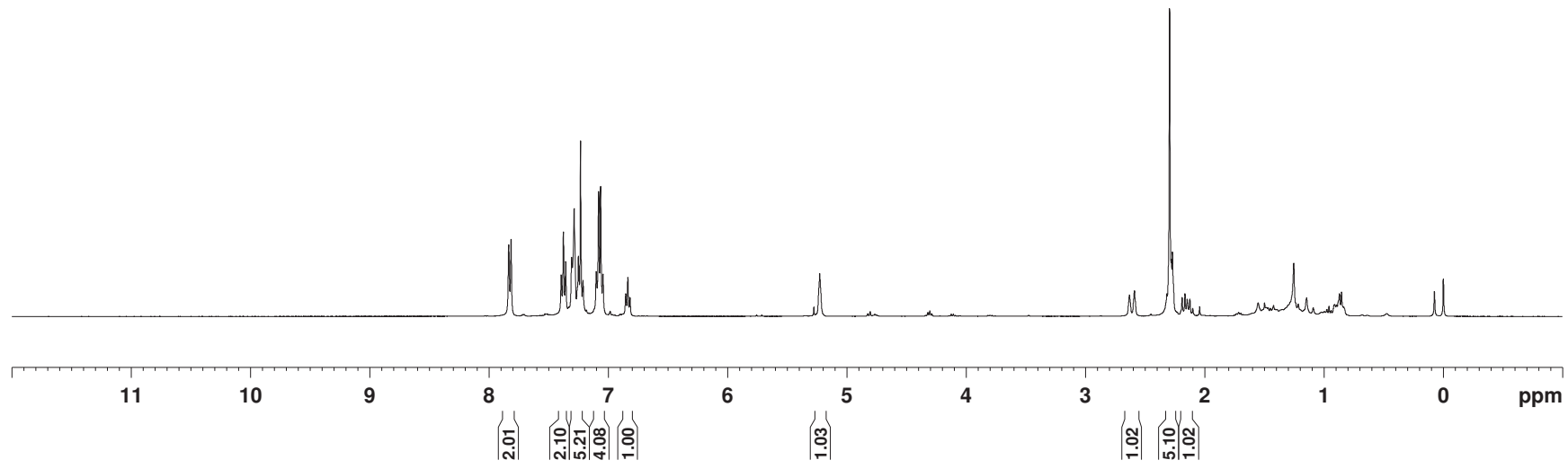<smiles>CCCC1CC([18OH])C(c2ccccc2)=NN1c1ccccc1</smiles> 
<smiles>O=[N+]([O-])O[P+]([O-])c1ccccc1</smiles>
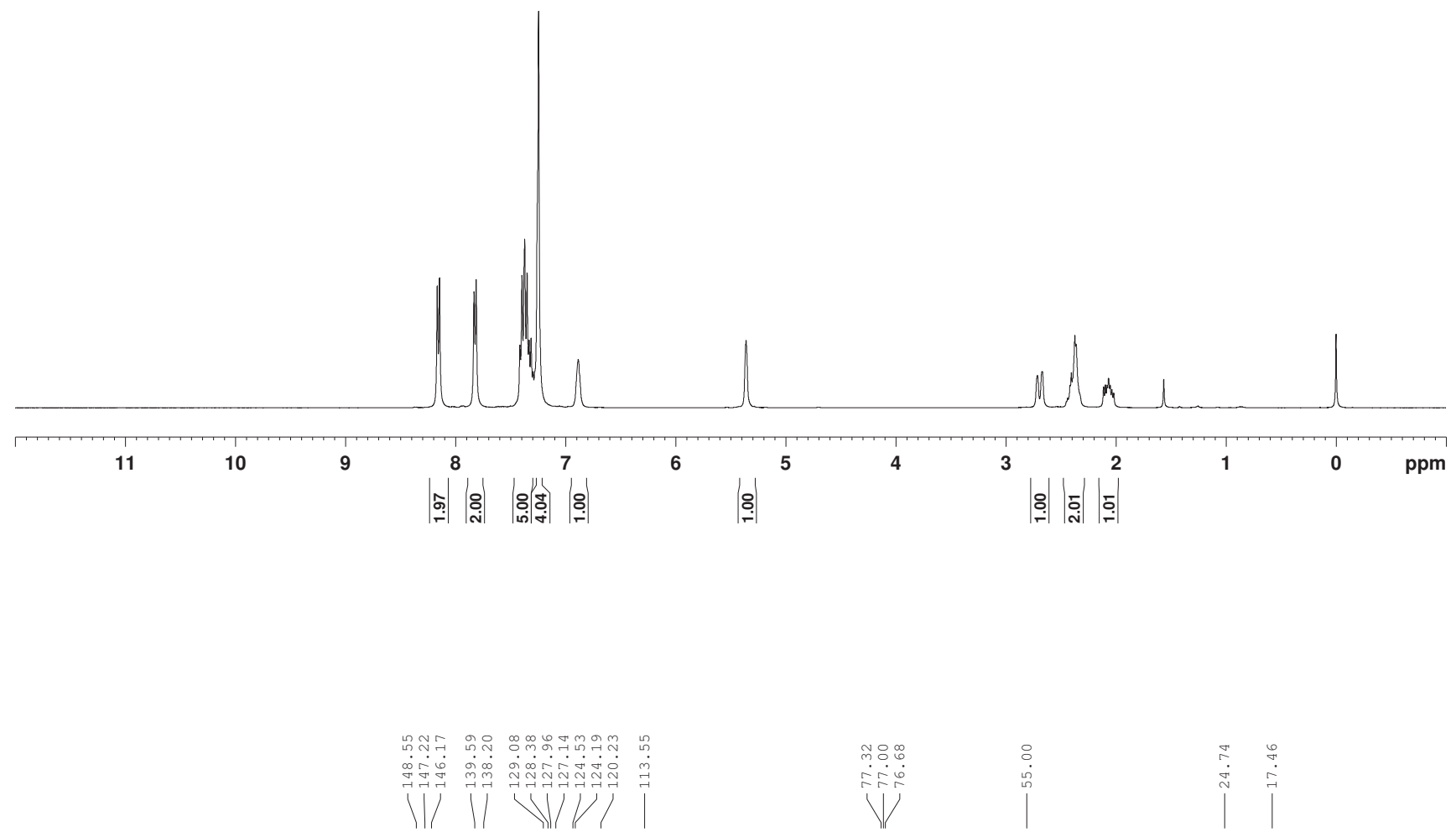<smiles>O=[N+]([O-])ON1CCC(c2ccccc2)=NN1c1ccccc1</smiles>

3aa

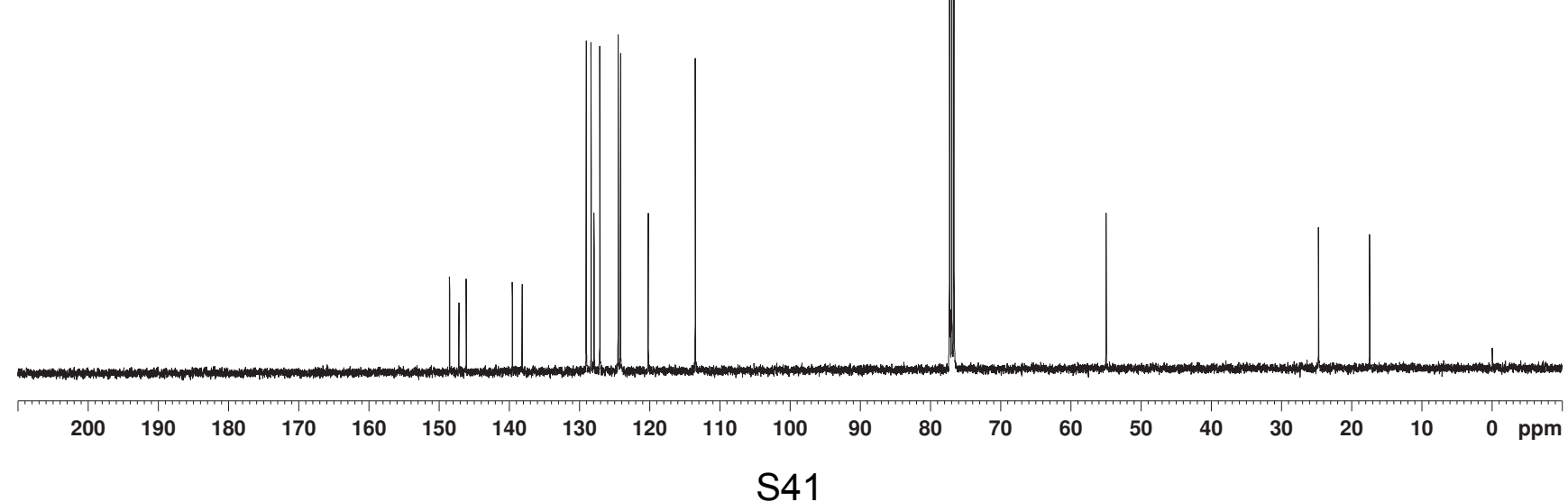


<smiles>c1ccc(C2=NN(c3ccccc3)C(c3ccccn3)CC2c2ccccc2)cc1</smiles>
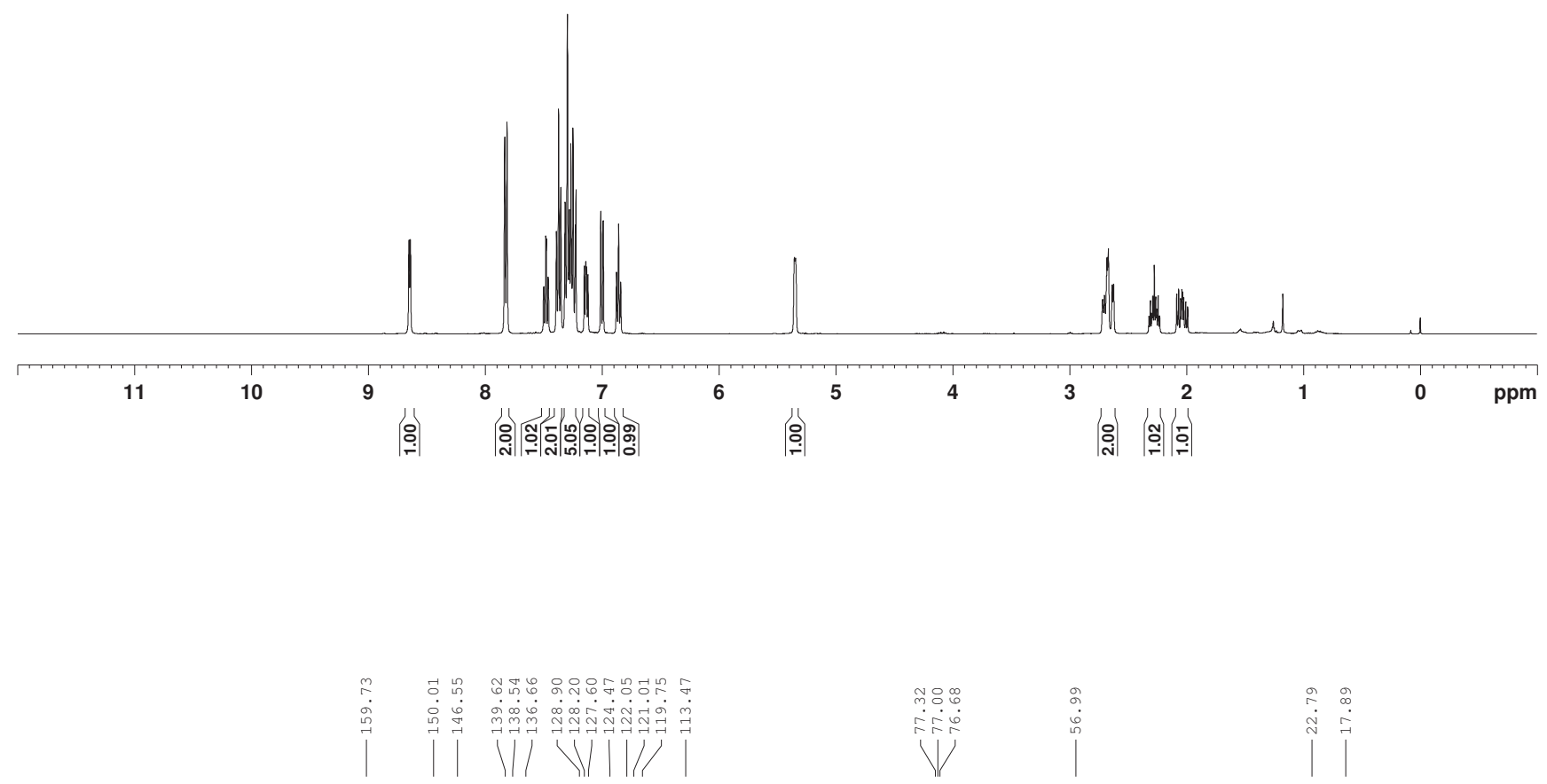<smiles>c1ccc(C2=NN(c3ccccc3)C(c3ccccn3)CC2c2ccccc2)cc1</smiles>

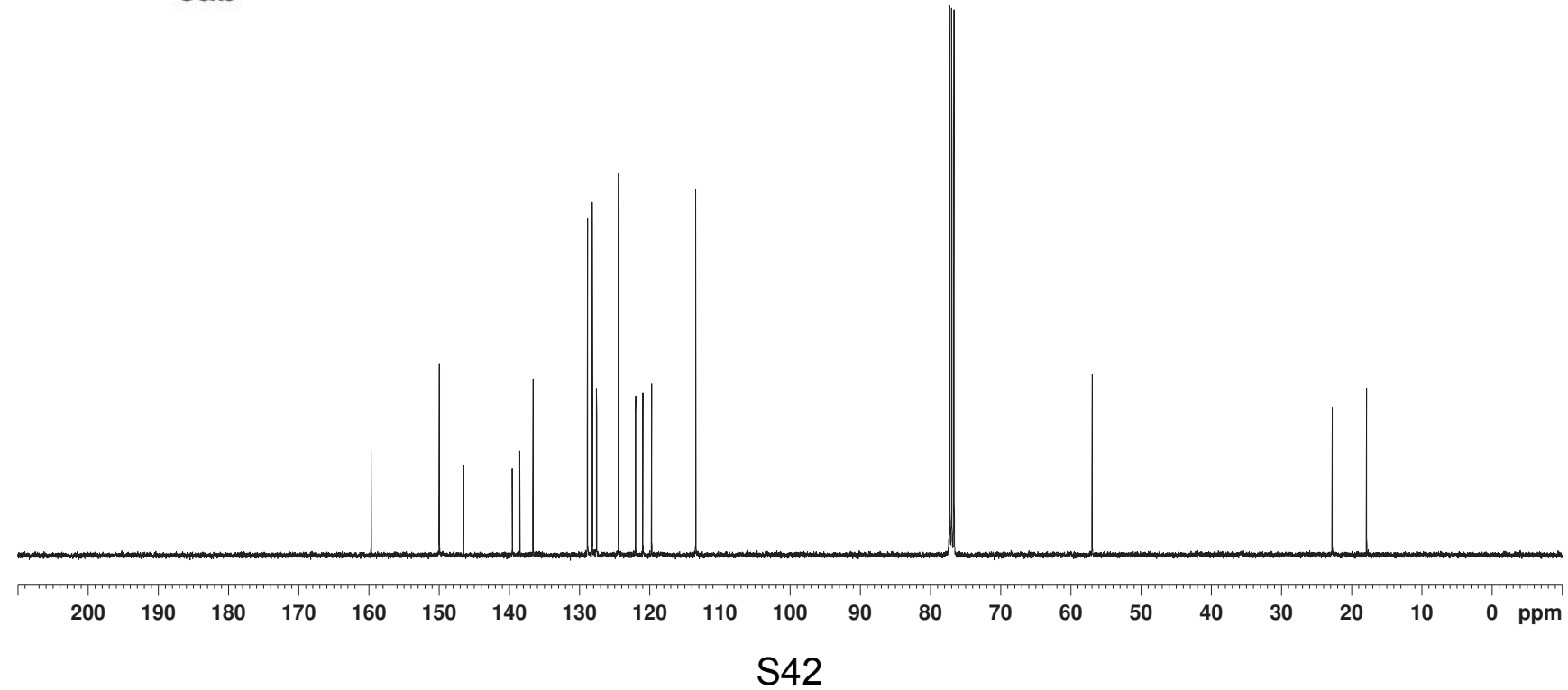



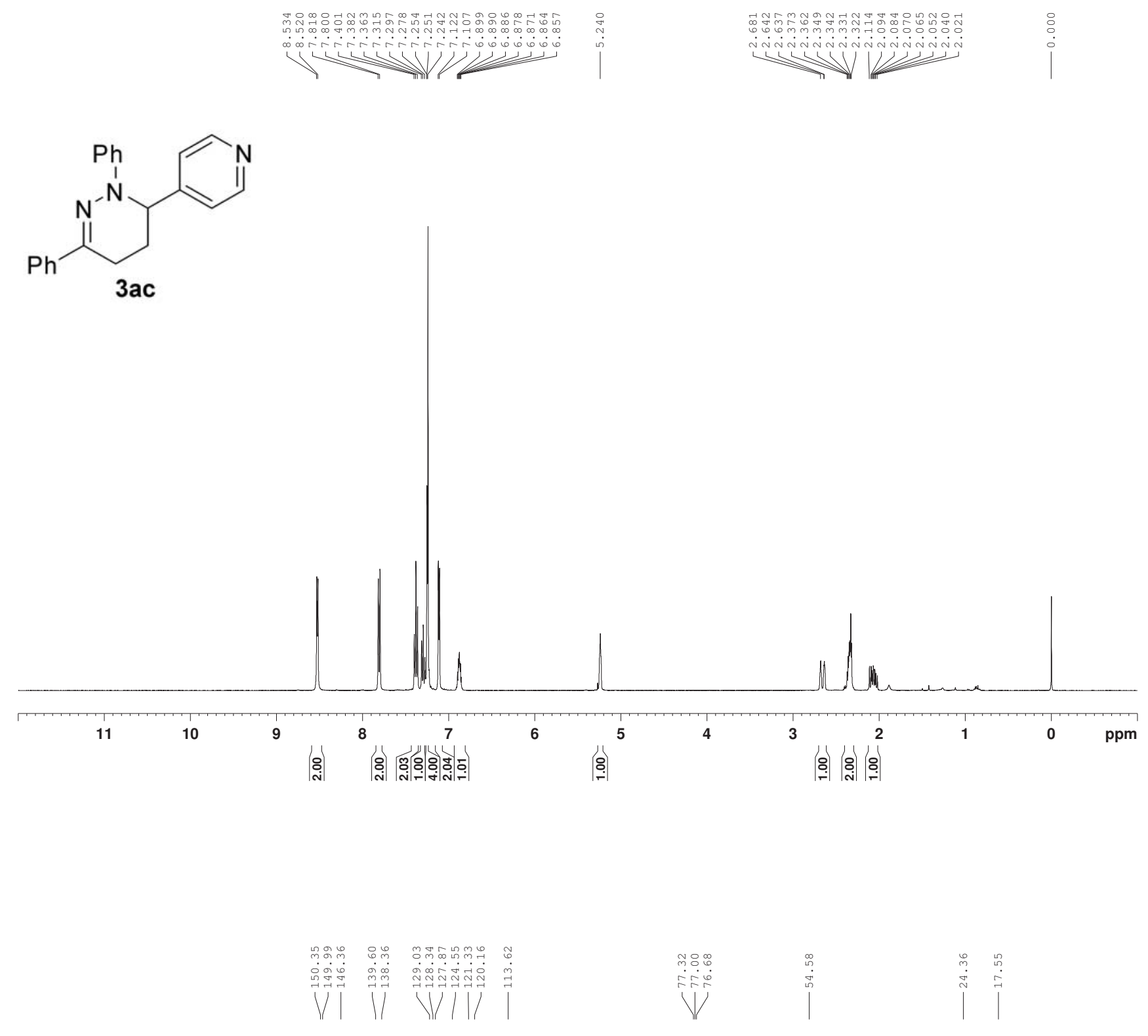<smiles>CC(C)(C)[14C]1CC(c2ccccc2)N(c2ccccc2)N(c2ccncc2)C1c1ccccc1</smiles>

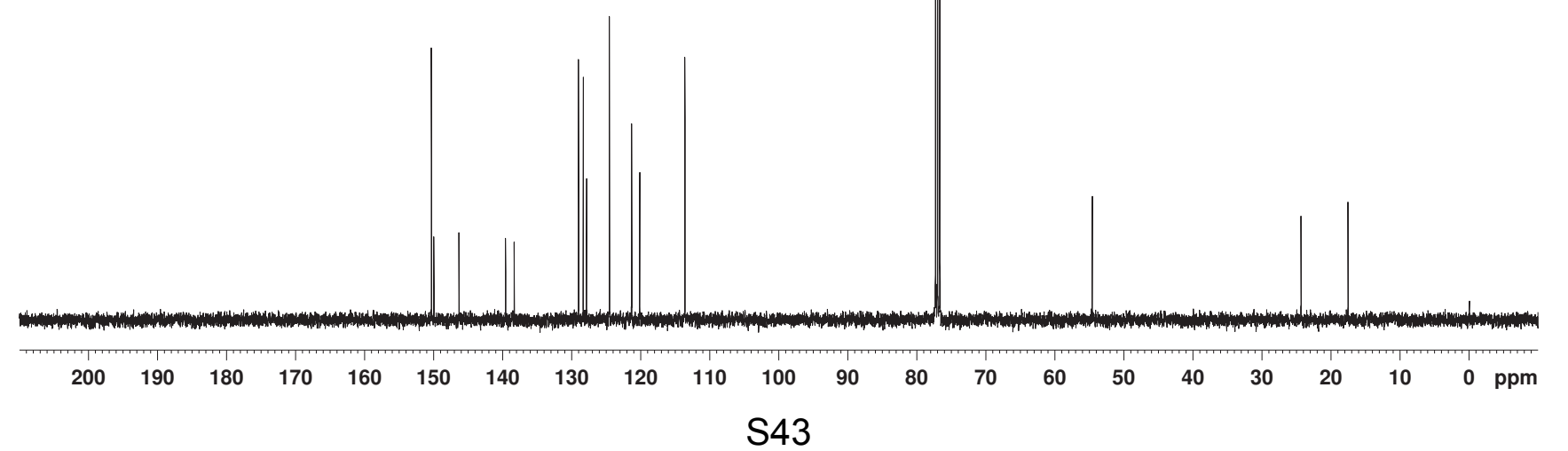


<smiles>CC(=O)C1(C)CCC(c2ccccc2)=NN1c1ccccc1</smiles>
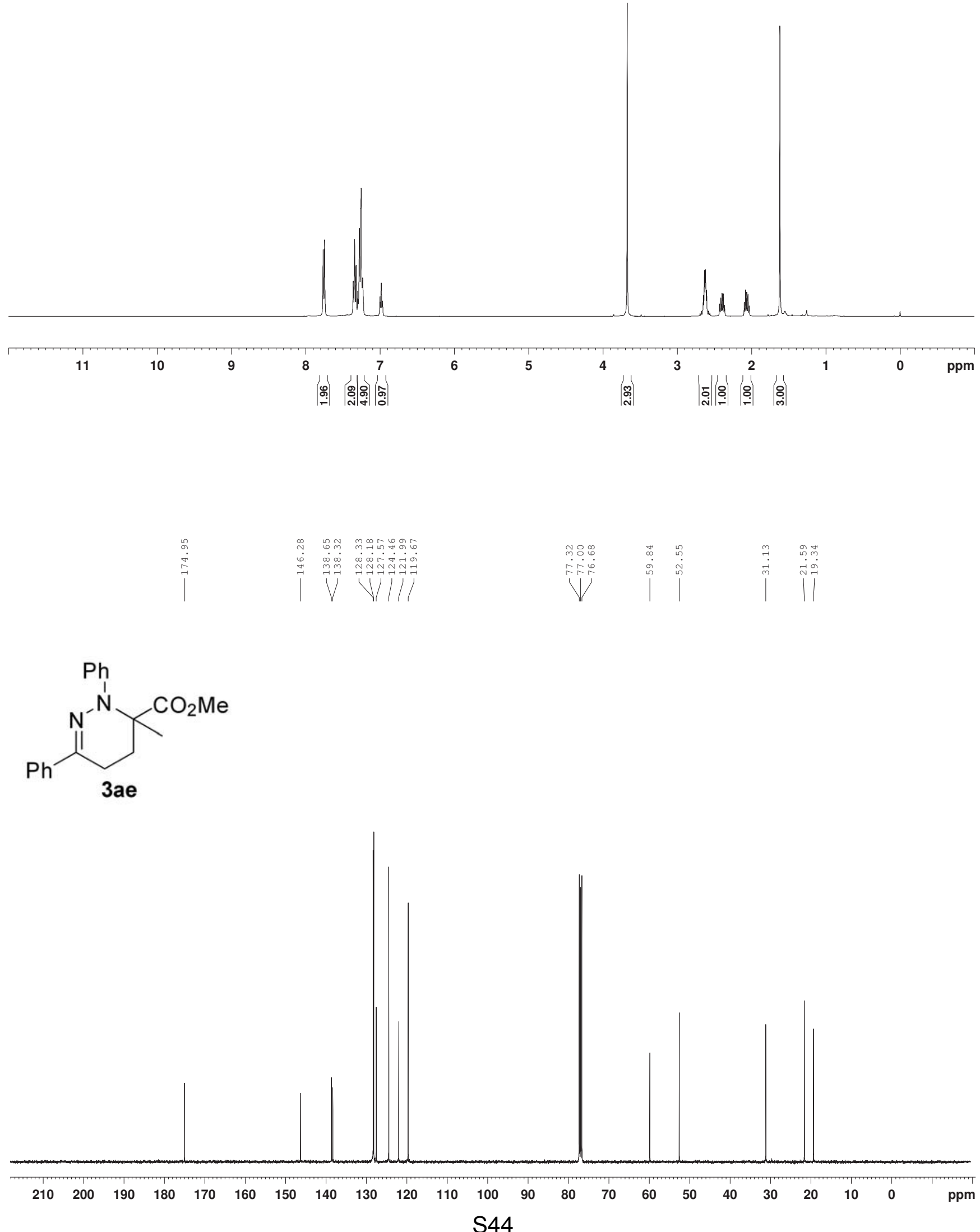
<smiles>CC1(c2ccccc2)CCC(c2ccccc2)=NN1c1ccccc1</smiles>

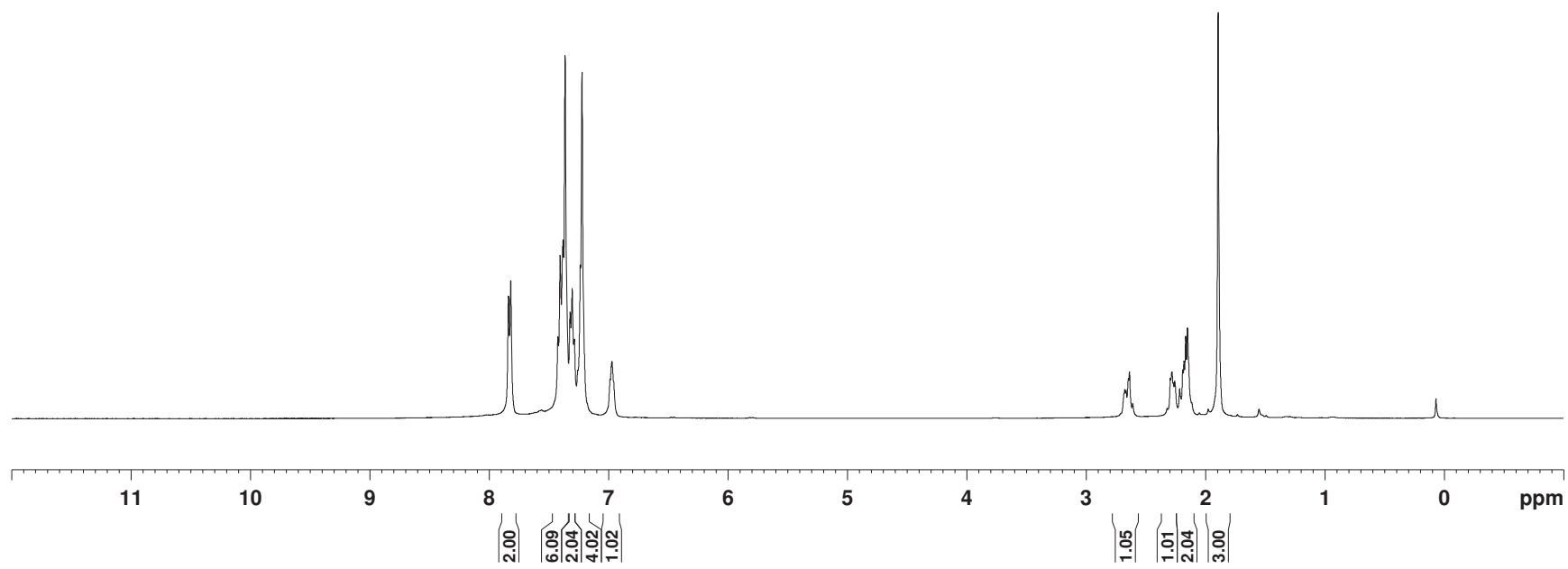<smiles>CC1(c2ccccc2)CCC(c2ccccc2)=NN1c1ccccc1</smiles>

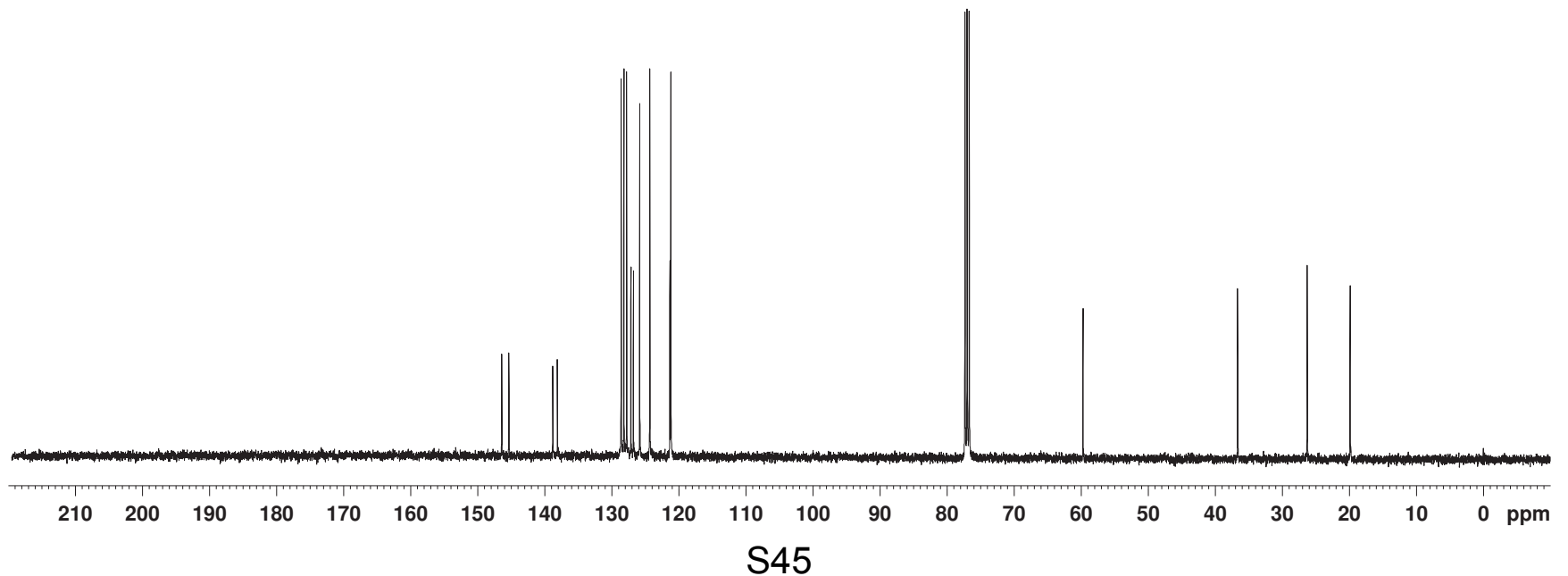

